Annales Geophysicae (2001) 19: 1065-1088 (c) European Geophysical Society 2001

\title{
Investigation of the outer and inner low-latitude boundary layers
}

\author{
T. M. Bauer ${ }^{1}$, R. A. Treumann ${ }^{1,2}$, and W. Baumjohann ${ }^{1,3}$ \\ ${ }^{1}$ Max-Planck-Institut für extraterrestrische Physik, Garching, Germany \\ ${ }^{2}$ Centre for Interdisciplinary Plasma Science, Garching, Germany \\ ${ }^{3}$ Institut für Weltraumforschung der Österreichischen Akademie der Wissenschaften, Graz, Austria
}

Received: 23 October 2000 - Revised: 26 June 2001 - Accepted: 2 July 2001

\begin{abstract}
We analyze 22 AMPTE/IRM crossings of the dayside low-latitude boundary layer for which a dense outer part can be distinguished from a dilute inner part. Whereas the plasma in the outer boundary layer (OBL) is dominated by solar wind particles, the partial densities of solar wind and magnetospheric particles are comparable in the inner boundary layer (IBL). For 11 events we find a reasonable agreement between observed plasma flows and those predicted by the tangential stress balance of an open magnetopause. Thus, we conclude that, at least in these cases, the OBL is formed by a local magnetic reconnection. The disagreement with the tangential stress balance in the other 11 cases might be due to reconnection being time-dependent and patchy. The northsouth component of the proton bulk velocity in the boundary layer is, on average, directed toward high latitudes for both low and high magnetic shear across the magnetopause. This argues clearly against the possibility that the dayside lowlatitude boundary layer is populated with solar wind plasma primarily from the cusps. "Warm", counterstreaming electrons that originate primarily from the magnetosheath and have a field-aligned temperature that is higher than the electron temperature in the magnetosheath by a factor of $1-5$, are a characteristic feature of the IBL. Profiles of the proton bulk velocity and the density of hot ring current electrons provide evidence that the IBL is on closed field lines. Part of the IBL may be on newly opened field lines. Using the average spectra of electric and magnetic fluctuations in the boundary layer, we estimate the diffusion caused by lower hybrid drift instability, gyroresonant pitch angle scattering, or kinetic Alfvén wave turbulence. We find that cross-field diffusion cannot transport solar wind plasma into the OBL or IBL at a rate that would account for the thickness $(\sim 1000 \mathrm{~km})$ of these sublayers. On the duskside, the dawn-dusk component of the proton bulk velocity in the IBL and magnetosphere is, on average, directed from the nightside toward local noon. Formation of the IBL may also be due to mechanisms operating in the magnetotail.
\end{abstract}

Correspondence to: R. A. Treumann (tre@mpe.mpg.de)
Key words. Magnetospheric physics (magnetopause, cusp and boundary layer; magnetospheath)

\section{Introduction}

Analyzing data of the ISEE satellites, Skopke et al. (1981) demonstrated that the low-latitude boundary layer earthward of the magnetopause on the flanks is often divided into two distinct parts. The outer part (called the "boundary layer proper" by Skopke et al., 1981) is filled with dense magnetosheath-like plasma moving tailward at speeds comparable to the magnetosheath flow. The hot, tenuous plasma in the inner part (Skopke et al., 1981, called it "halo") is a mixture of solar wind and magnetospheric particles and it is moving slowly like the plasma in the magnetosphere proper. A similar structure of the flank boundary layer was reported by Fujimoto et al. (1998a). They pointed out that the flow in the inner part, which they called the "mixing region", was directed sunward. From this, they concluded that the plasma in the mixing region is most likely on closed field lines and that the solar wind particles seen in this region do not enter locally. Instead, the mixing might take place farther down the tail and the solar wind particles in the mixing region might be supplied to the magnetosphere by the same process as those in the cold dense plasma sheet (Fujimoto et al., 1998b), which often fills a large portion of the magnetotail.

At the dayside magnetopause, observations of a lowlatitude boundary layer containing magnetosheath-like plasma in its outer parts and hot, tenuous plasma in its inner parts, respectively, have been presented by Song et al. $(1990,1993)$ and Le et al. (1996) for the northward interplanetary magnetic field. Hapgood and Bryant (1990), Hall et al. (1991), and Nakamura et al. (2000) also found a similar structure for other directions of the interplanetary magnetic field. For a recent review of the bulk properties of the boundary layer see Hultqvist et al. (1999).

In our companion paper (Bauer et al., 2000, hereafter referred to as Paper I), we analyzed two dayside magnetopause 
crossings for which we could also make a clear distinction between a dense outer boundary layer (OBL) and a dilute inner boundary layer (IBL). While the OBL is clearly dominated by solar wind plasma, the partial densities of solar wind and magnetospheric particles are comparable in the IBL. For the crossing on 17 September 1984, the magnetic shear across the magnetopause was $15^{\circ}$ and reconnection signatures were absent. On 21 September 1984, the magnetic shear was $90^{\circ}$ and we found clear evidence for local magnetic reconnection. On 21 September 1984, the plasma in the OBL is accelerated due to reconnection and on 17 September 1984 , it is moving somewhat slower than the magnetosheath flow. In contrast, the plasma flow in the IBL is stagnant on 21 September 1984 and even directed back to the subsolar point on 17 September 1984. For both events, the drop in the density of ring current electrons from the IBL to the OBL suggests that the interface between both sublayers is a topological boundary related to reconnection.

In fact, most researchers agree that the OBL is formed by magnetic reconnection. Numerous observations of reconnection signatures (e.g. Sonnerup et al., 1981; Gosling et al., 1990; Fuselier et al., 1991; Phan et al., 2000, Paper I) provide strong evidence that the OBL is either on open field lines or on field lines that have been first opened by reconnection, then filled with solar wind plasma, and closed later on (Nishida, 1989).

Less is known about the formation of the IBL. The stagnant (partly sunward directed) flow in the IBL, the balance of particle flux parallel and antiparallel to the magnetic field, $\boldsymbol{B}$, and the drop in the density of ring current electrons from the IBL to the OBL all suggest that the IBL is on closed field lines. If this is true, it still remains to be explained how the solar wind particles observed in the IBL have been transported onto these closed field lines. One possible transport mechanism is cross-field diffusion due to the wave-particle interaction. In order to assess the importance of diffusion, we will compute the average wave spectra in Sect. 6 and derive estimates of average diffusion coefficients.

Le et al. (1996) explained the formation of the OBL and IBL for the northward interplanetary magnetic field in terms of reconnection poleward of the cusps in both hemispheres. In their model, the OBL is on open field lines that have been formed by reconnection between magnetosheath field lines and lobe field lines poleward of one cusp. The IBL is identified on closed field lines that have become closed by the reconnection of the open end of the field lines at the other cusp.

Lockwood (1997) suggested that the inner part of the lowlatitude boundary layer is not on closed field lines, but on newly opened field lines. If reconnection is in a steady state, solar wind particles entering the boundary layer do not fill the entire region of open field lines between the magnetopause and the separatrix, but only fill the region bounded by the magnetopause and an inner edge that depends on the fieldaligned velocity, $v_{\|}^{\prime}$, of the particles in the de HoffmannTeller frame (e.g. Gosling et al., 1990). Within the time elapsed since a field line was opened by reconnection, a point on this field line located earthward of the inner edge cannot be reached by particles flying at velocity $v_{\|}^{\prime}$. Since bulk velocities and thermal speeds of solar wind ions are much smaller than thermal speeds of solar wind electrons, the inner edge of the ions is closer to the magnetopause than the inner edge of the electrons. In this picture, the OBL might be the region bounded by the magnetopause and the ion edge, and the IBL might be the region of newly opened field lines between the ion and electron edges. The absence of solar wind ions would explain why the flow in the IBL is not coupled to the flow in the magnetosheath and OBL. The "counterstreaming" or "warm" electrons observed in the IBL (e.g. Ogilvie et al., 1984; Hall et al., 1991; Pottelette and Treumann, 1998, Paper I) would be solar wind electrons earthward of the ion edge.

For the statistical analysis of reconnection signatures in Paper I, we selected a data set of 40 magnetopause crossings from all dayside magnetopause passes of the AMPTE/IRM spacecraft. In this paper, we will use the same data set (1) to survey the plasma populations in the OBL, the IBL, and the neighboring magnetosphere (Sect. 4), (2) to perform a superposed epoch analysis of the magnetopause, of the interface between the OBL and IBL, and the inner edge of the boundary layer (Sect. 5), and (3) to examine the average wave activity (Sect. 6). In Sect. 7, we will use our results to discuss the formation of the boundary layer.

\section{Instrumentation}

We use measurements of the triaxial flux gate magnetometer (Lühr et al., 1985), the plasma instrument, and the wave instrument on board the IRM spacecraft. The plasma instrument (Paschmann et al., 1985) consists of two electrostatic analyzers of the top hat type, one for ions and one for electrons. Three-dimensional distributions with 128 angles and 30 energy channels in the energy-per-charge range from $15 \mathrm{~V}$ to $30 \mathrm{kV}$ for electrons, and $20 \mathrm{~V}$ to $40 \mathrm{kV}$ for ions, were obtained every satellite rotation period, i.e. every $4.4 \mathrm{~s}$. From each distribution, microcomputers within the instruments computed moments of the distribution functions of ions and electrons: densities in three contiguous energy bands: the bulk velocity vector, the pressure tensor, and the heat flux vector. In these computations, it was assumed that all the ions were protons. Whereas the moments were transmitted to the ground at the full time resolution, the distributions themselves were transmitted less frequently because the allocated telemetry was limited. The ELF/VLF spectrum analyzer of the IRM wave experiment package (Häusler et al., 1985) used the signal from the $47 \mathrm{~m}$ tip-to-tip dipole antenna to provide a relatively coarse frequency resolution, yet rapid temporal resolution with essentially continuous coverage of electric wave signals from $25 \mathrm{~Hz}$ to $250 \mathrm{kHz}$. 


\section{Data set and occurrence of step like profiles}

We studied all IRM passes through the dayside (08:00-16:00 LT) magnetopause region for which the relevant data are available: magnetometer measurements, plasma moments at spin resolution, ion and electron distribution functions of the full energy-per-charge range, and electric wave spectra. The statistical data set analyzed in this paper and Paper I contains 40 magnetopause crossings which were obtained by selecting all crossings that occured during the aforementioned dayside passes and that fulfilled the following criteria: (1) The crossing is a complete crossing from the magnetosheath to the magnetosphere proper (or vice versa); (2) The duration, $\Delta t_{\mathrm{BL}}$, of the boundary layer is at least $30 \mathrm{~s}$; (3) At least two electron distribution functions are measured in the boundary layer; (4) The time intervals in the magnetosheath before (after) the boundary layer and the time intervals in the magnetosphere after (before) the boundary layer are so long that an unambiguous identification of the magnetopause and the earthward edge of the boundary layer is possible.

Criteria 2 and 3 are required in order to resolve the internal structure of the boundary layer, i.e. to distinguish gradual time profiles from step like profiles, whereas sharp steps in the profiles may mark topological boundaries or discontinuities associated with reconnection; gradual transitions are expected for a boundary layer formed by diffusion.

How exactly do we define the magnetopause and the inner edge of the boundary layer? Examples that illustrate the way we define the magnetopause and the inner edge are given by the case studies in Paper I. Progressing from the magnetosheath to the magnetosphere, we define the inner edge of the boundary layer as the point where solar wind electrons or warm electrons disappear. In previous studies of IRM data (Paschmann et al., 1993; Phan and Paschmann, 1996), the magnetopause was identified with the rotation of the magnetic field in the case of high shear crossings and with a change in the thermal properties of the plasma in the case of low shear crossings. Since defining the magnetopause in a different manner for low and high shear might introduce some bias into the statistical analysis, we avoid defining the magnetopause with magnetic field data. Instead, we identify the magnetopause both for high and low shear as a change in the distribution functions of solar wind ions and electrons. Although this change is theoretically not well understood, it is well established by observations.

What kind of temporal profiles are observed for the boundary layer crossings included in the data set? Examining the time series of the total density, we divide the 40 crossings into four classes: for 22 crossings, two plateaus of the density can be distinguished. One plateau has a density comparable to the magnetosheath density and is identified as an OBL. The other plateau has a distinctly lower density and is identified as an IBL. Therefore, we obtain 22 crossings for which the boundary layer can be divided into an OBL and IBL (class 1). For 6 of the 40 crossings we observe only a highdensity plateau and identify this as an OBL (class 2). For 2 of the 40 crossings we observe only a low-density plateau and identify this as an IBL (class 3 ). The remaining 10 crossings (class 4) do not show any plateaus or pronounced steps in the boundary layer and are classified as crossings with gradual profiles.

For the superposed epoch analyses of Sect. 5, the interface between OBL and IBL is used as a key time. There are crossings in our data set during which IRM moves back and forth between the OBL and IBL. In this case, only the first (last) OBL and last (first) IBL of an inbound (outbound) crossing is used for the superposed epoch analyses.

All crossings occurred near the equatorial plane at latitudes less than $30^{\circ}$. We will distinguish between low and high magnetic shear. For low (high) shear crossings, the angle, $\left|\Delta \varphi_{B}\right|$, between the magnetic fields in the magnetosheath and in the magnetosphere is less (greater) than $40^{\circ}$. Furthermore, we will distinguish between "Walén events" and "non-Walén events". The identification of crossings as Walén or non-Walén events was done in Paper I, where we checked if the plasma moments measured across the magnetopause satisfy the tangential stress balance (Walén relation)

$\boldsymbol{V}_{p}^{\prime}=\boldsymbol{V}_{p}-\boldsymbol{V}_{\mathrm{HT}}= \pm \boldsymbol{c}_{A}$

of a rotational discontinuity. Here, $\boldsymbol{V}_{\boldsymbol{p}}$ is the proton bulk velocity, $\boldsymbol{V}_{\text {HT }}$ is the velocity of the de Hoffmann-Teller frame, $\boldsymbol{V}_{p}^{\prime}$ is the proton bulk velocity in the de Hoffmann-Teller frame, and $\boldsymbol{c}_{A}$ is the Alfvén velocity. The $+\operatorname{sign}(-\operatorname{sign})$ is valid when the normal component $V_{p n}$ of the proton bulk flow has the same (opposite) direction as $B_{n}$.

The first step in the identification of Walén events was to test if a de Hoffmann-Teller frame exists. This was done by inspecting scatter plots of the convection electric field, $-\boldsymbol{V}_{p} \times \boldsymbol{B}$, versus the transformation electric field, $-\boldsymbol{V}_{\mathrm{HT}} \times$ $B$, and computing the corresponding correlation and regression coefficients. In the second step, we tested Eq. (1) by inspecting scatter plots of $\boldsymbol{V}_{p}^{\prime}$ versus $\boldsymbol{c}_{A}$ and computing the corresponding correlation and regression coefficients.

We found for 11 of the 22 selected IRM crossings that have both an OBL and IBL, a linear relation

$\boldsymbol{V}_{p}^{\prime}=\Lambda \boldsymbol{c}_{A}$

is fulfilled, with $\Lambda$ as a constant coefficient. We considered this as a "reasonable agreement with the Walén relation" and refer to these crossings as Walén events. The pros and cons of the validity of this relation have been discussed in detail in Paper I. For non-Walén events, the linear relation (2) cannot be satisfied. The apparent $50 \%$ agreement of magnetopause crossings with the above relation cannot be taken as proof, however, that the magnetopause would be a rotational discontinuity for half of the time. This number is accidental due to the systematic uncertainties involved. However, the cases when such a relation exists in spite of the large uncertainties in the measurement supports the view that in such cases, a de Hoffmann-Teller frame exists and this signals that the magnetopause has, on average, the character of a rotational discontinuity. 
Table 1. Occurrence rate of plasma populations in the OBL, IBL, and magnetosphere proper, respectively

\begin{tabular}{lccc}
\hline & OBL & IBL & Sphere \\
\hline solar wind ions & $100 \%$ & $100 \%$ & $0 \%$ \\
solar wind/warm electrons & $100 \%$ & $100 \%$ & $0 \%$ \\
cold ions & $14 \%$ & $45 \%$ & $59 \%$ \\
cold electrons & - & - & $73 \%$ \\
ring current ions & $100 \%$ & $100 \%$ & $100 \%$ \\
ring current electrons & $100 \%$ & $100 \%$ & $100 \%$ \\
\hline
\end{tabular}

Most of the slopes $\Lambda$ that we determined by fitting the data to Eq. (2) are less in magnitude than the value of unity predicted for a magnetohydrodynamic rotational discontinuity. This discrepancy was also found in numerous previous studies (e.g. Paschmann et al., 1986; Sonnerup et al., 1990; Phan et al., 1996; Scudder et al., 1999). Reasons for this discrepancy have been discussed in these publications and in Paper I.

The duration, $\Delta t_{\mathrm{BL}}$, of the examined boundary layer crossings varies between $30 \mathrm{~s}$ and $70 \mathrm{~min}$. The arithmetic average of $\Delta t_{\mathrm{BL}}$ for the 40 crossings is $530 \mathrm{~s}$ and the geometric average is $210 \mathrm{~s}$. For the 24 high shear crossings, the arithmetic (geometric) average of $\Delta t_{\mathrm{BL}}$ is $440 \mathrm{~s}(160 \mathrm{~s})$. For the 16 low shear crossings, the arithmetic (geometric) average of $\Delta t_{\mathrm{BL}}$ is $670 \mathrm{~s}$ (300 s). Average boundary layer durations of IRM crossings were also calculated by Phan and Paschmann (1996). They found an arithmetic (geometric) average of $74 \mathrm{~s}$ (48 s) for high shear and an arithmetic (geometric) average of 206 s (90 s) for low shear. Since Phan and Paschmann (1996) did not sort out crossings with $\Delta t_{\mathrm{BL}}<30 \mathrm{~s}$, it is not surprising that their averages are lower than ours.

From now on, we consider only the 22 crossings of class 1 , since we expect that the distinction between the OBL and IBL provides additional insight into the physics of the boundary layer. The arithmetic (geometric) average of the duration, $\Delta t_{\mathrm{OBL}}$, of the OBL is $160 \mathrm{~s}(70 \mathrm{~s})$. The arithmetic (geometric) average of the duration, $\Delta t_{\mathrm{IBL}}$, of the IBL is $290 \mathrm{~s}(80 \mathrm{~s})$. Hence, typical durations of the OBL and IBL are comparable to each other.

\section{Survey of plasma populations}

In the dayside low-latitude boundary layer and the neighboring regions of the magnetosheath and magnetosphere, the following plasma populations can be distinguished: solar wind plasma with thermal energies of $\sim 400 \mathrm{eV}$ for the ions and $\sim 50 \mathrm{eV}$ for the electrons, hot ring current particles with energies of 1-100 keV, and cold particles of ionospheric origin with thermal energies of several $\mathrm{eV}$. We identify the different populations by inspecting the plots of the distribution functions.

The cold particles may either reach the magnetopause region directly from the ionosphere along magnetic field lines or they may be supplied by convection from the plasmasphere (e.g. Fuselier et al., 1989, 1997; Elphic et al., 1996; Borovsky et al., 1997). During the magnetopause passes analyzed in Paper I, we also observed "warm" electrons. These are typical for the IBL and they have a field-aligned temperature higher than the electron temperature in the magnetosheath by a factor of $1-5$.

Hall et al. (1991) concluded that the warm, counterstreaming electrons are solar wind electrons on closed field lines. Ogilvie et al. (1984) suggested that they are beams from the ionosphere that reach the low-latitude boundary layer along $\boldsymbol{B}$. Figure 1 presents one-dimensional cuts through the distributions measured on 17 September 1984. Two-dimensional cuts through the same distributions were provided in Fig. 7a of Paper I. Let us first have a look at the phase space density, $f_{e}$, in a cut along $\boldsymbol{B}$ (left diagram). Typical field-aligned velocities, $v_{\|}$, of the warm electrons are $6000-15000 \mathrm{~km} / \mathrm{s}$. In this range, the value of $f_{e}\left(v_{\|}, v_{\perp}=0\right)$ in the IBL (solid line) is comparable to that in the OBL (long dashed line). On the other hand, the phase space density of the cold ionospheric electrons in the magnetosphere (dash-dotted line) falls below the detection threshold for $v_{\|}>6000 \mathrm{~km} / \mathrm{s}$ and is thus at least one order of magnitude lower than the phase space density of the warm electrons. If the warm electrons also originated from the ionospher, e the high phase space density for $v_{\|}>6000 \mathrm{~km} / \mathrm{s}$ could only be explained as the consequence of field-aligned acceleration on field lines mapping to the IBL. Field-aligned beams of ionospheric electrons accelerated upward are indeed observed at low altitudes on auroral field lines (e.g. Lundin et al., 1987; Boehm et al., 1995; Carlson et al., 1998). Those upgoing electrons may form part of the population of warm electrons. However, the relatively sharp boundary between the IBL and the magnetosphere argues against the possibility that the ionosphere is the main source of the warm electrons. Any mechanism should not only explain the acceleration of ionospheric electrons upward at low altitudes, but also why the acceleration merely maps to the limited region of field lines connected to the IBL. The fact that the curves of $f_{e}\left(v_{\|}, v_{\perp}=0\right)$ in the IBL and OBL are pretty close to one another in the range of 6000 $15000 \mathrm{~km} / \mathrm{s}$ suggests that the electrons in the IBL and OBL have a common source: the shocked solar wind plasma of the magnetosheath. For $v_{\|}<6000 \mathrm{~km} / \mathrm{s}$, the phase space density in the IBL is considerably less than that in the OBL. This indicates that the mechanism which transports solar wind electrons into the IBL does not work effectively for electrons with small field-aligned velocities. This is also reflected in the diagram for $f_{e}\left(v_{\|}=0, v_{\perp}\right)$. For $v_{\|} \approx 0$, the phase space density in the IBL is not very different from that in the magnetosphere proper.

Table 1 gives the occurrence rates of the different particle populations in the OBL, the IBL, and the adjacent magnetosphere. Hot ring current particles are always observed in all three regions. Solar wind electrons or warm electrons are always observed in the OBL and IBL, respectively, and they are never observed in the magnetosphere proper. Solar wind ions are always observed in the OBL and never in the 

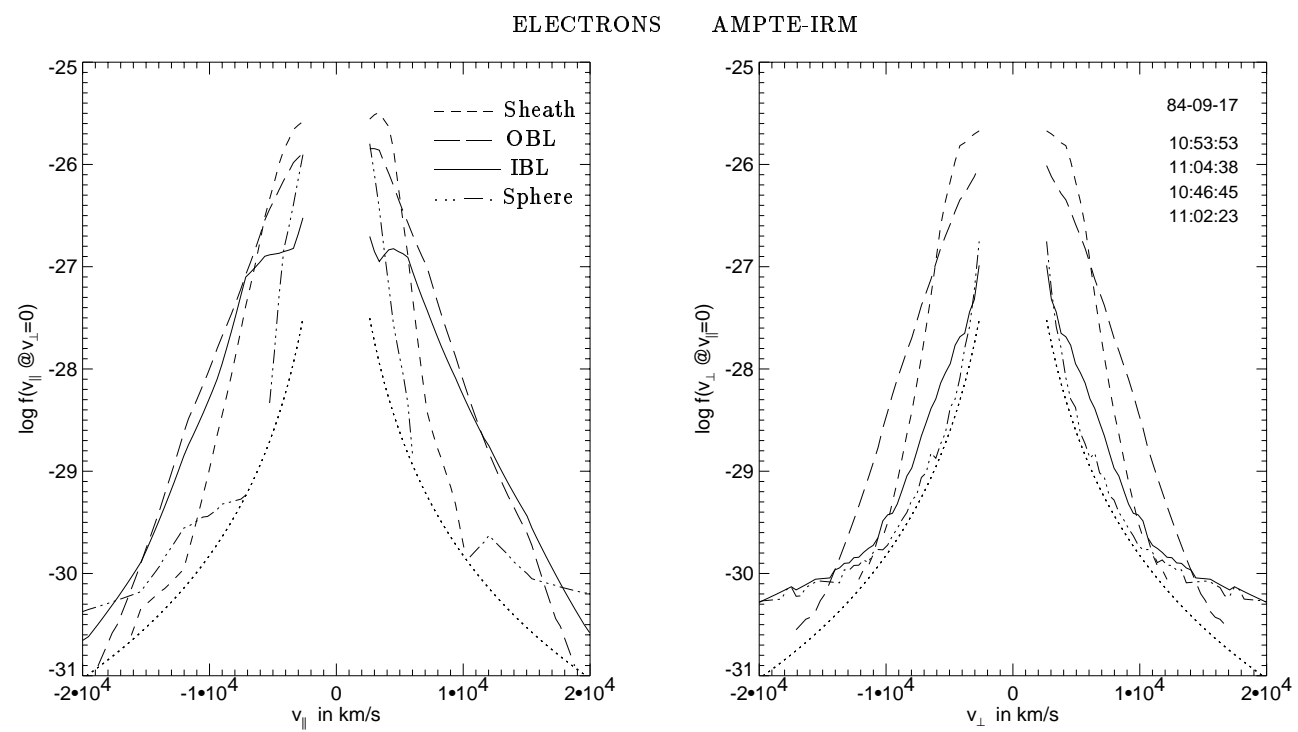

Fig. 1. One-dimensional cuts through the electron distributions measured on 17 September 1984. The measurements are taken in the magnetosheath at 10:46:45 (short dashed line), in the outer boundary layer at 10:53:53 (long dashed line), in the inner boundary layer at 11:02:23 (solid line), and in the magnetosphere proper at 11:04:38 (dash-dotted line). The left diagram shows the phase space density $f_{e}\left(\right.$ in $\mathrm{cm}^{-6} \mathrm{~s}^{3}$ ) as a function of $v_{\|}$for $v_{\perp}=0$, and the right diagram shows $f_{e}$ as a function of $v_{\perp}$ for $v_{\|}=0$. The dotted line gives the detection threshold.

magnetosphere. Many distribution functions measured in the IBL exist that do not show solar wind ions, such as the distributions measured on 21 September 1984 before the flux transfer event (Paper I). However, for each of the 22 magnetopause crossings, there exists at least one distribution measured in the IBL that does show solar wind ions. This is what the value of $100 \%$ means in Table 4.

The occurrence rate of cold ionospheric ions increases from the OBL toward the magnetosphere. We did not compute the occurrence rate of cold electrons in the boundary layer, since at this location the phase space density of cold electrons is often masked by the phase space density of the dominating solar wind or warm electrons. In the magnetosphere proper, cold electrons are detected for 16 of the 22 crossings. In the duskside (13:00-16:00 LT) magnetosphere, cold electrons are more frequently detected ( 7 out of 8 crossings) than in the dawnside (08:00-11:00 LT) magnetosphere (5 out of 10). For the cold ions we did not find a local time dependence. Checking $D_{\text {st }}$ and $A E$ indices, we did not find any correlation between the occurrence of cold particles and these indices.

\section{Superposed epoch analysis}

Superposed epoch analyses of IRM magnetopause crossings were performed by Paschmann et al. (1993), Phan et al. (1994), and Phan and Paschmann (1996). While these studies focussed on the magnetopause, we are particularly interested in the interface between the OBL and the IBL and the inner edge of the boundary layer. In Sect. 5.1, average profiles of the 7 boundary layer crossings earthward of a low shear magnetopause are compared with average profiles of the 15 boundary layer crossings earthward of a high shear magnetopause. Of the 22 crossings selected for the superposed epoch analysis, 11 are Walén events. In Sect. 5.2, we compare the average profiles of the 11 Walén events with those of the 11 non-Walén events. Finally, in Sect. 5.3, average profiles of the 13 crossings dawnward of local noon are compared with the average profiles of the 9 crossings duskward of local noon. The magnetic field and the proton bulk velocity are displaced in $L M N$ boundary normal coordinates (Russell and Elphic, 1979).

\subsection{Low versus high magnetic shear}

Figures 2 and 3 present a superposed epoch analysis of the 7 IRM crossings with low magnetic shear $\left(\left|\Delta \varphi_{B}\right|<40^{\circ}\right)$ and the 15 crossings with high magnetic shear $\left(\left|\Delta \varphi_{B}\right|>40^{\circ}\right)$. For our analysis, we use two key times: the time when the magnetopause is crossed and the time when the interface between the OBL and the IBL is crossed. The time of the magnetopause crossing is set to zero and the order of the time series is reversed for outbound crossings. Next, we normalize the time axis so that all OBLs have the same normalized duration. Thus, normalized time $\hat{t}=0$ corresponds to the magnetopause and normalized time $\hat{t}=1$ corresponds to the interface between the OBL and the IBL for each event. Finally, the traces of each parameter as a function of the normalized time $\hat{t}$ are superposed and averaged.

The panels of Figs. 2 and 3 include data from $\hat{t}=-1$ to $\hat{t}=2$. This means that we use all measurements in the magnetosheath that are obtained with less than one OBL in duration, $\Delta t_{\mathrm{OBL}}$, sunward of the magnetopause and all measurements that are obtained with less than $\Delta t_{\mathrm{OBL}}$ earthward of the interface between the OBL and the IBL. The data are 
Low Magnetic Shear
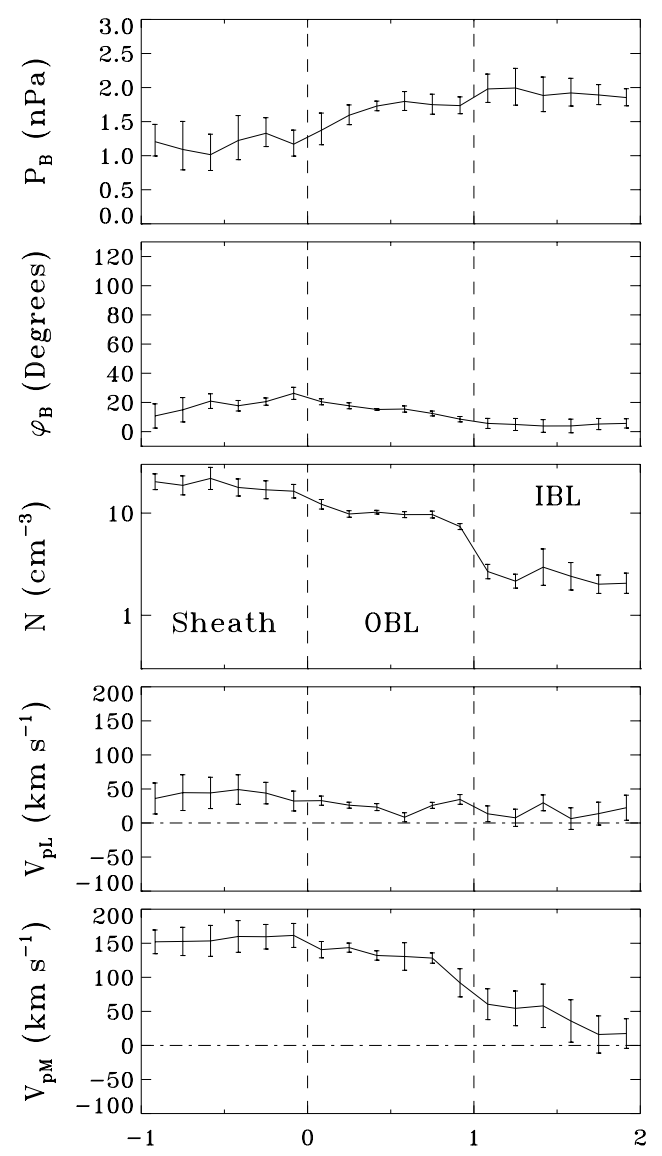

Normalized Time
High Magnetic Shear
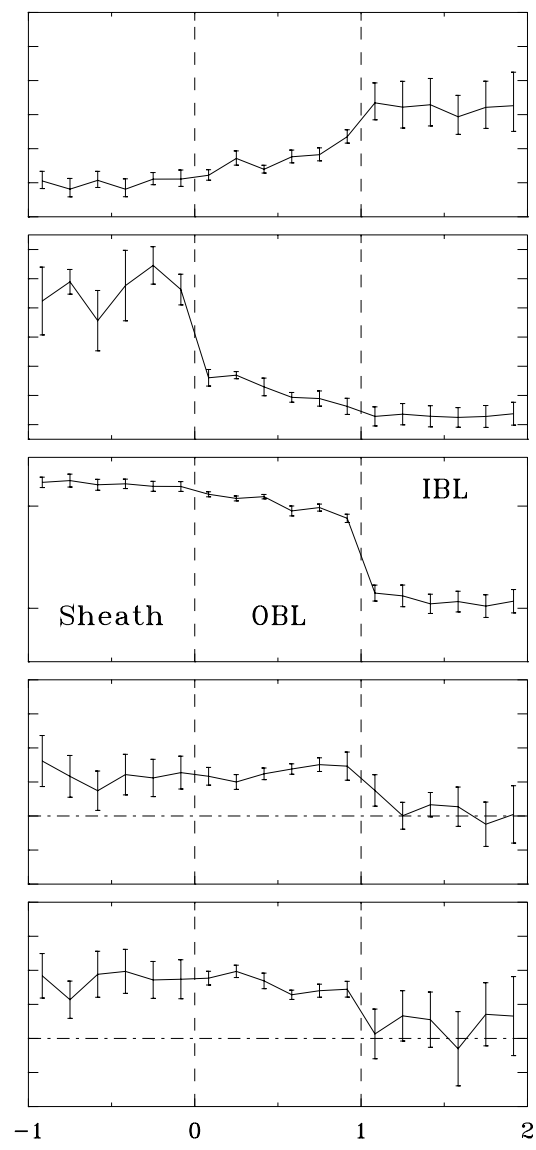

Fig. 2. Superposed epoch analysis of the magnetic pressure, $P_{B}$, the magnetic field rotation angle, $\varphi_{B}$, the total plasma density, $N$, and the components $V_{p L}, V_{p M}$ of the proton bulk velocity across the outer boundary layer. The sign of $V_{p L}$ is reversed for crossings at southern latitudes and the sign of $V_{p M}$ is reversed for crossings duskward of local noon. The left panels display data from 7 low shear crossings and the right panels display data from 15 high shear crossings. Normalized time $\hat{t}=0$ corresponds to the magnetopause and normalized time $\hat{t}=1$ corresponds to the interface between the OBL and the IBL. Vertical error bars indicate the standard deviation of the average of each parameter.

sorted into 18 bins and averaged within each bin. Parameters that can change sign are averaged linearly, and parameters that are positive by definition are averaged logarithmically. The density ratios $N_{0 p} / N_{p}$ and $N_{0 e} / N_{e}$, which are averaged linearly, represent an exception.

The length of the error bars in Figs. 2 and 3 gives the standard deviation of the average, i.e. the standard deviation, $\sigma$, of the respective parameter or its logarithm divided by the square root of the number, $n$, of events. Since the absolute value of some parameters may vary considerably from case to case, the time average in the OBL of each parameter or its logarithm is subtracted from the individual values before the averaging over events. After the event averaging, the event average of all time averages in the OBL is added again. This procedure has only a minor influence on the event averages plotted in Figs. 2 and 3, but it reduces the error bars. The plotted error bars are useful in judging whether or not the variation within one profile is significant. However, they cannot be used to judge whether or not the absolute value of a parameter in the panels on the left side is significantly different from its absolute value in the panels on the right side of Figs. 2 and 3.

Let us start with Fig. 2. The trace of $\varphi_{B}$ in the second panel shows the rotation of the magnetic field tangential to the magnetopause. The sign of $\varphi_{B}$ is reversed for events for which the change $\Delta \varphi_{B}$ from the magnetosphere to the magnetosheath is negative. This is done in order to avoid that events with clockwise rotation compensate for events with an anticlockwise rotation. As expected from the respective definition of low and high shear, $\varphi_{B}$ changes only slightly across the low shear boundary layer, whereas it rotates, on average, by about $90^{\circ}$ across the high shear boundary layer. About $70 \%$ of the change in $\varphi_{B}$ occurs from the bins sheathward of the magnetopause to the first bin in the OBL. The remaining rotation is accomplished within the OBL.

The third panel of Fig. 2 shows clearly that the interface between the OBL and the IBL is the location where most of the density decrease from the magnetosheath level of about $20 \mathrm{~cm}^{-3}$ to the magnetospheric level of about $2 \mathrm{~cm}^{-3}$ takes place. Paschmann et al. (1993) and Phan et al. (1994) showed that for low shear, a plasma depletion layer evolves in the magnetosheath adjacent to the magnetopause. In the plasma depletion layer the magnetic field piles up and the plasma is squeezed out. Since ions with high field-aligned velocities leave the plasma depletion layer earlier than ions with low field-aligned velocities, the solar wind ions in the plasma 
Low Magnetic Shear
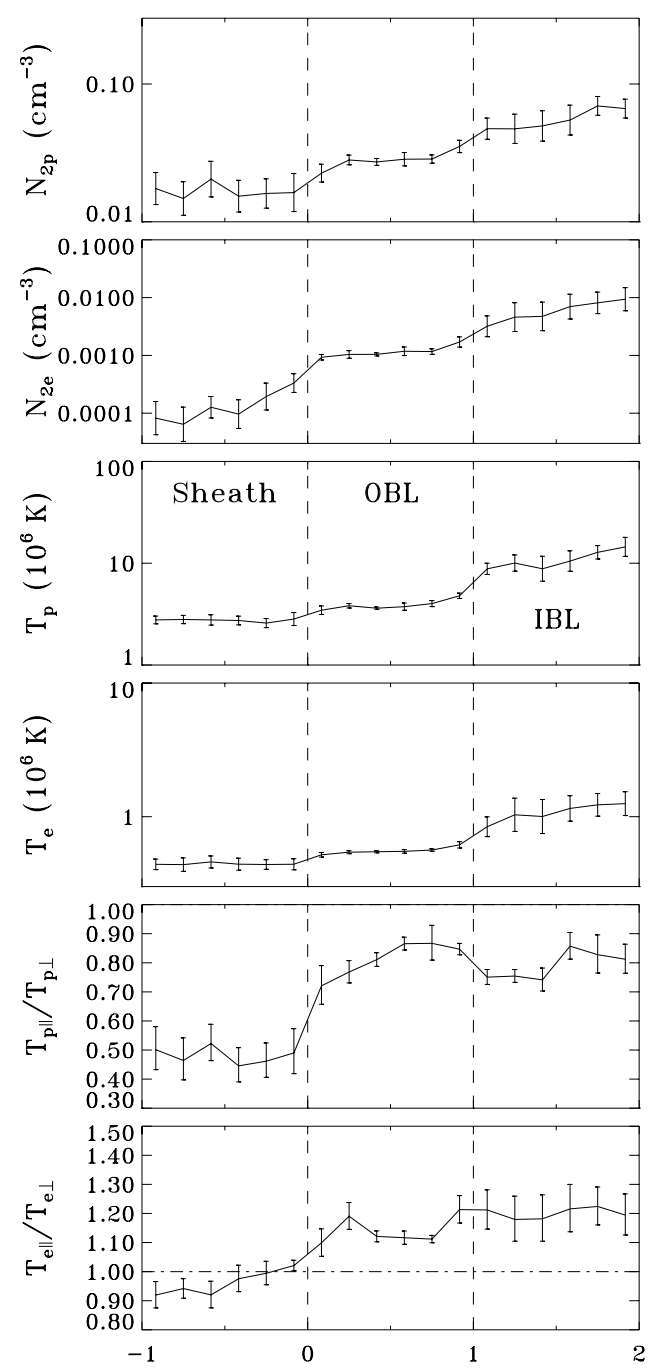

High Magnetic Shear
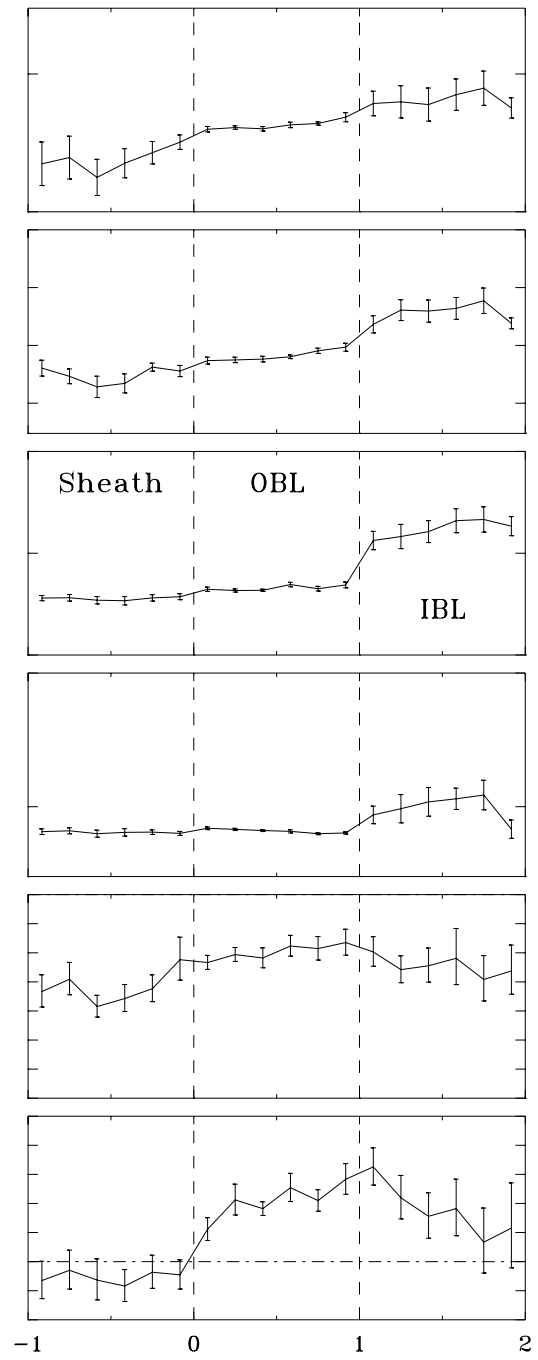

Normalized Time

Fig. 3. Superposed epoch analysis of and the partial density, $N_{2 p}$, of protons above $8 \mathrm{keV}$, and the partial density, $N_{2 e}$, of electrons above $1.8 \mathrm{keV}$, the proton temperature, $T_{p}$, the electron temperature, $T_{e}$, the proton temperature anisotropy, $T_{p \|} / T_{p \perp}$, and the electron temperature anisotropy, $T_{e \|} / T_{e \perp}$, across the outer boundary layer. The format is the same as in Fig. 2. depletion layer exhibit a strong perpendicular temperature anisotropy. For our events, the average density in front of the low shear magnetopause is not lower than in front of the high shear magnetopause. However, the first panel of Fig. 2 shows that the magnetic pressure, $P_{B}$, in front of the low shear magnetopause is higher by more than a factor of 2 due to its pile up in the plasma depletion layer. Moreover, the proton temperature anisotropies displayed in the fifth panel of Fig. 3 are clearly different for low and high shear. Although $T_{p \|} / T_{p \perp}$ is about 0.7 in front of the high shear magnetopause it is less than 0.5 in front of the low shear magnetopause. In the OBL, $T_{p \|} / T_{p \perp}$ is about 0.8 both for high and low shear.

The upper two panels of Fig. 3 give the partial density, $N_{2 p}$, of protons above $8 \mathrm{keV}$, which is dominated by ring current ions, and the partial density, $N_{2 e}$, of electrons above $1.8 \mathrm{keV}$, which is dominated by ring current electrons. Confirming the case studies of Paper I, we find that $N_{2 e}$ drops from the IBL to the OBL. A further reduction of $N_{2 e}$ is ob- served from the OBL to the magnetosheath. For low shear this reduction is much more pronounced than for high shear. This might indicate that for high shear the plasmas on both sides of the magnetopause mix faster. $N_{2 p}$ also decreases both from the IBL to the OBL and from the OBL to the magnetosheath.

The last two panels of Fig. 2 display the components $V_{p L}$ and $V_{p M}$ of the proton bulk velocity. The sign of $V_{p L}$ is reversed for crossings at southern latitudes so that $V_{p L}>0$ corresponds to a poleward flow away from the equatorial plane. The sign of $V_{p M}$ is reversed for crossings duskward of local noon so that $V_{p M}>0$ corresponds to a tailward flow toward the flanks. The plasma flow in the magnetosheath and boundary layer is directed poleward and toward the flanks. The magnitudes of $V_{p L}$ and $V_{p M}$ are roughly the same in the magnetosheath and in the OBL, but they drop clearly at the interface between the OBL and the IBL. Looking at the error bars, we find that for most of the bins in the inner boundary 
Walen Relation
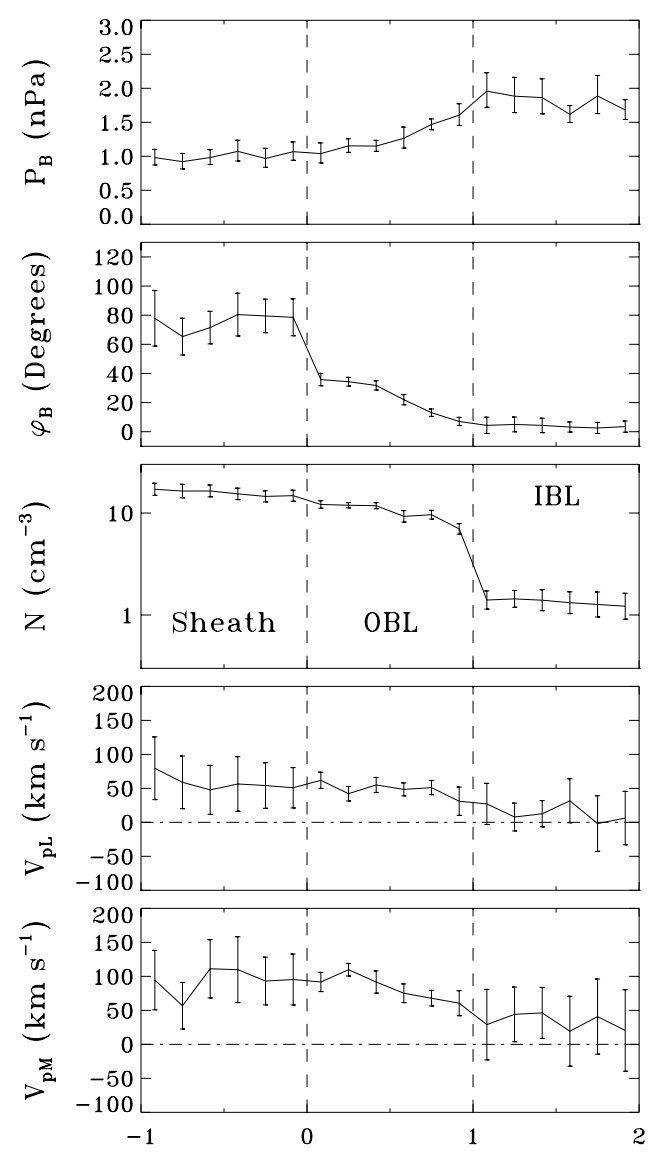

Normalized Time
No Walen Relation
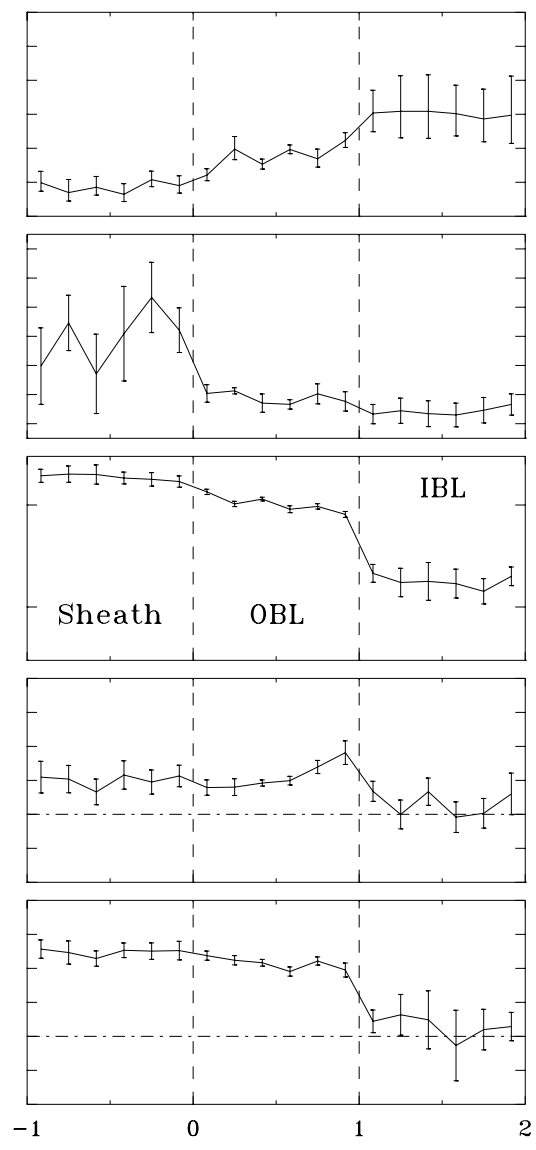

Fig. 4. Superposed epoch analysis of the magnetic pressure, $P_{B}$, the magnetic field rotation angle, $\varphi_{B}$, the total plasma density, $N$, and the components $V_{p L}, V_{p M}$ of the proton bulk velocity across the outer boundary layer. The sign of $V_{p L}$ is reversed for crossings at southern latitudes and the sign of $V_{p M}$ is reversed for crossings duskward of local noon. The left panels display data from 11 crossings that show reasonable agreement with the Walén relation, and the right panels display data from 11 crossings without agreement. Normalized time $\hat{t}=0$ corresponds to the magnetopause and normalized time $\hat{t}=1$ corresponds to the interface between the OBL and the IBL. Vertical error bars indicate the standard deviation of the average of each parameter.

layer $V_{p L}$ and $V_{p M}$ are not significantly different from zero.

The proton and electron temperatures displayed in Fig. 3 exhibit a slight increase at the magnetopause and a strong increase from the OBL to the IBL. On average, the thermal energy, $K T_{p}\left(K T_{e}\right.$ ), of the protons (electrons) is about $500 \mathrm{eV}(60 \mathrm{eV})$ in the OBL and about $1.5 \mathrm{keV}(100 \mathrm{eV})$ in the IBL. In the last panel of Fig. 3, we can see that the electrons show a perpendicular temperature anisotropy in the magnetosheath, while in both parts of the boundary layer fieldaligned anisotropies, $T_{e \|} / T_{e \perp} \approx 1.2$, are observed.

\subsection{Walén events versus non-Walén events}

Now we use the same data intervals as in Sect. 5.1 and plot the averages of all Walén events against the averages of all non-Walén events in Figs. 4 to 6 . The magnetic pressure, $P_{B}$, decreases from the IBL to the OBL. Whereas it is about constant across the magnetopause for the Walén events, it changes by a factor of 2 across the magnetopause for the non-Walén events. This reflects the finding of Paschmann et al. (1986) and Phan et al. (1996) that for low plasma $\beta$ in the magnetosheath agreement with the Walén relation is more frequently observed than for high $\beta$. Here $\beta$ is the ratio $P / P_{B}$ of the plasma pressure, $P=N_{p} K T_{p}+N_{e} K T_{e}$, and magnetic pressure. Since the average of $P$ is about the same for the Walén and non-Walén events, the different averages of $P_{B}$ correspond to different averages of $\beta$. The plasma $\beta$ in the magnetosheath adjacent to the magnetopause is on average 0.9 for the Walén events and 2 for the non-Walén events.

The north-south component, $V_{p L}$, of the proton bulk velocity is approximately $50 \mathrm{~km} / \mathrm{s}$ in the magnetosheath and OBL, and the dawn-dusk component, $V_{p M}$, is approximately $100 \mathrm{~km} / \mathrm{s}$. The averages of both velocity components drop at the interface between OBL and IBL. This drop is more pronounced for the non-Walén events. This indicates that the flow in the IBL is not coupled to the flow in the magnetosheath and OBL, irrespective of whether or not agreement with the Walén relation is fulfilled. The superposed epoch traces of $N, N_{2 p}, N_{2 e}, T_{p}, T_{e}, T_{p \|} / T_{p \perp}$, and $T_{e \|} / T_{e \perp}$ do not show any remarkable differences between Walén and nonWalén events.

The upper two diagrams of Fig. 6 serve to illustrate the degree of agreement between the Walén relation (1) and the plasma moments measured in the vicinity of the magnetopause. The parameters plotted are the ratio, $V_{p \|}^{\prime} / c_{A}$, of the field-aligned component of the proton bulk velocity in the de Hoffmann-Teller frame and the Alfvén speed, and the angle, 


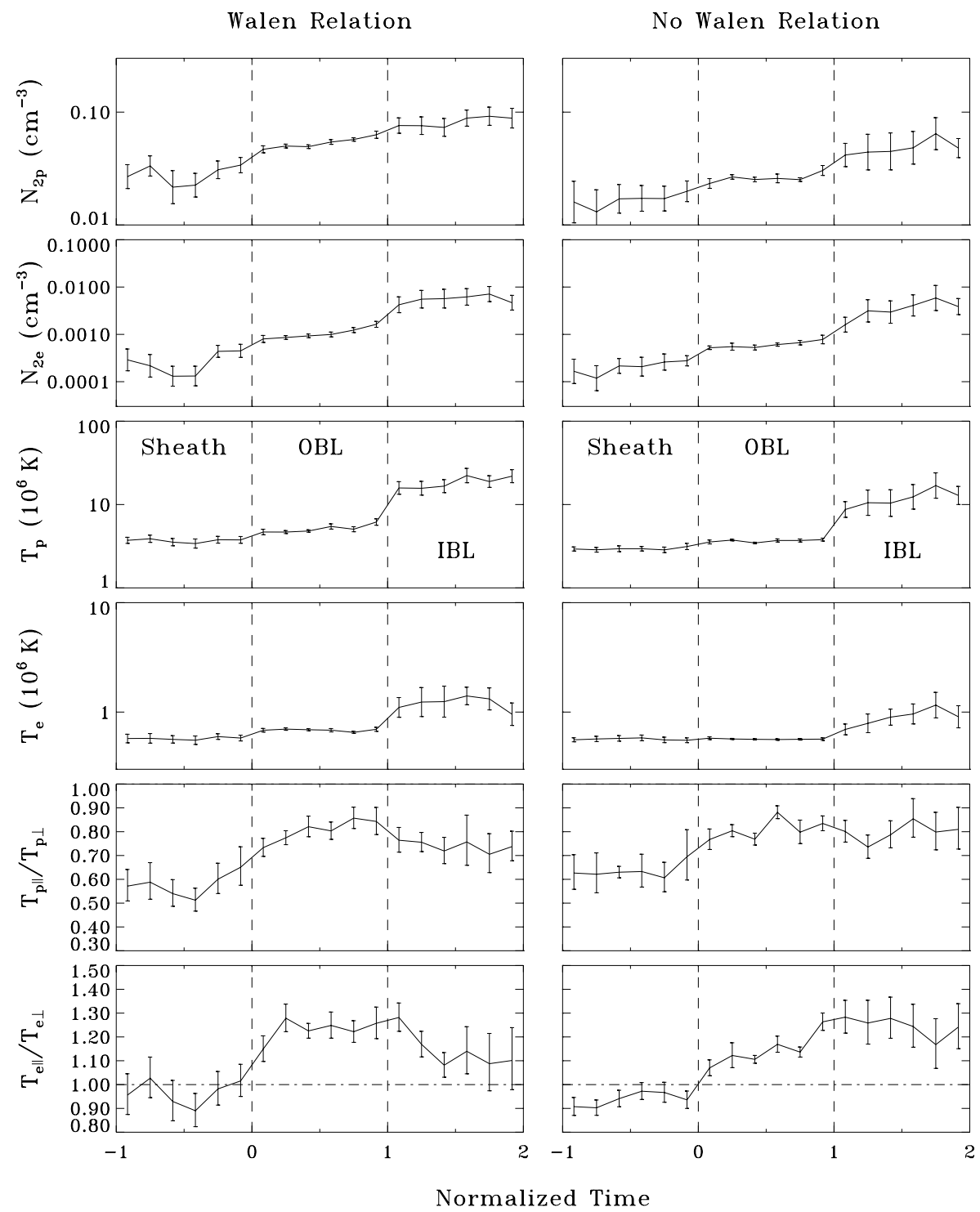

Fig. 5. Superposed epoch analysis of the partial density, $N_{2 p}$, of protons above $8 \mathrm{keV}$, the partial density, $N_{2 e}$, of electrons above $1.8 \mathrm{keV}$, the proton temperature, $T_{p}$, the electron temperature, $T_{e}$, the proton temperature anisotropy, $T_{p \|} / T_{p \perp}$, and the electron temperature anisotropy, $T_{e \|} / T_{e \perp}$, across the outer boundary layer. The format is the same as in Fig. 4.
$\theta_{V^{\prime} B}$, between the proton bulk velocity in the de HoffmannTeller frame and the magnetic field direction. Since these parameters are only useful if a well-defined de Hoffmann-Teller frame exists and if the sign of $B_{n}$ is known, they are not plotted for the non-Walén events. For magnetopause crossings for which the test of the Walén relation indicates $B_{n}>0$, the sign of $V_{p \|}^{\prime}$ is reversed and $\theta_{V^{\prime} B}$ is replaced by $180^{\circ}-\theta_{V^{\prime} B}$. Thus, $V_{p \|}^{\prime} / c_{A}$ should, according to the theory of a rotational discontinuity be equal to +1 , and $\theta_{V^{\prime} B}$ should be equal to $0^{\circ}$ on open field lines in the vicinity of the magnetopause.

The average of $\theta_{V^{\prime} B}$ for all Walén events is less than $30^{\circ}$ in the normalized time interval $-1<\hat{t}<0$ and approximately $30^{\circ}$ for the first five bins in the OBL. This shows that the plasma flow in the de Hoffmann-Teller frame is indeed predominantly field-aligned. In view of the fact that a onedimensional time stationary rotational discontinuity can only be a crude model of the real magnetopause, it is not surprising that the average angles $\theta_{V^{\prime} B}$ are not closer to $0^{\circ}$. In the
IBL, the plasma flow in the de Hoffmann-Teller frame is, on average, nearly perpendicular to $B$, which indicates that the IBL is either on closed field lines or on newly opened field lines.

The ratio $V_{p \|}^{\prime} / c_{A}$ is between 0.6 and 1 in the normalized time interval $-1<\hat{t}<0$ and approximately 0.4 for the first five bins in the OBL. The deviation from the theoretical value $V_{p \|}^{\prime} / c_{A}=1$ reflects the finding of Paper I that the slope $\Lambda$ in Eq. (2) is, in general, less than 1. In the IBL, the average of $V_{p \|}^{\prime} / c_{A}$ falls below 0.1 .

While the superposed epoch traces of $V_{p \|}^{\prime} / c_{A}$ and $\theta_{V^{\prime} B}$ measure the quality of the fit to Eq. (1), the last panel of Fig. 6 presents a result that is independent of the test of the Walén relation, vis-a-vis, a superposed epoch analysis of the proton heat flux, $H_{p \|}$, parallel to $\boldsymbol{B}$. For Walén events with $B_{n}>0$, the sign of $H_{p \|}$ is reversed before the superposition. Thus, $H_{p \|}<0$ indicates heat flux that is opposed to the proton bulk velocity in the de Hoffmann-Teller frame and there- 
Walen Relation
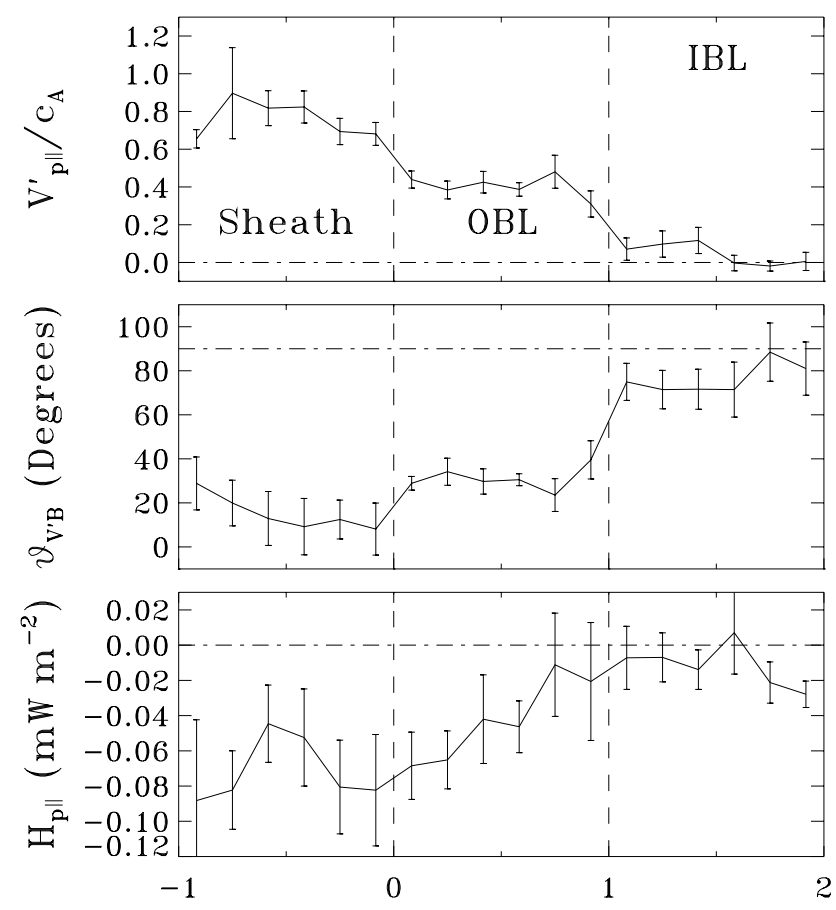

Normalized Time

Fig. 6. Superposed epoch analysis of the ratio, $V_{p \|}^{\prime} / c_{A}$, of the fieldaligned component of the proton bulk velocity in the de HoffmannTeller frame and the Alfvén speed, the angle, $\vartheta_{V^{\prime} B}$, between the proton bulk velocity in the de Hoffmann-Teller frame and the magnetic field direction, and the proton heat flux, $H_{p \|}$, parallel to $\boldsymbol{B}$ across the outer boundary layer. For magnetopause crossings for which the test of the Walén relation indicates $B_{n}>0$, the sign of $V_{p \|}^{\prime}$ and $H_{p \|}$ is reversed. Thus, $\vartheta_{V^{\prime} B}$ is replaced by $180^{\circ}-\vartheta_{V^{\prime} B}$ in this case. The format is the same as in Fig. 4.

fore directed outward along open field lines. The superposed epoch average of $H_{p \|}$ reveals that there is indeed an outward directed proton heat flux of the order of $0.05 \mathrm{~mW} / \mathrm{m}^{2}$ in the magnetosheath and OBL. Ion distribution functions associated with heat flux have been examined in Paper I.

We do not plot the routinely computed electron heat flux, since it is often strongly affected by an instrumental defect (see Appendix 1 of Paschmann et al., 1986). Therefore, the electron heat flux can best be studied by direct inspection of the distribution functions (Paper I).

\subsection{Dawnside versus duskside}

Figures 7 and 8 compare crossings on the morning side (08:00-12:00 LT) with magnetopause crossings on the evening side (12:00-16:00 LT). Whereas the superposed epoch traces of the dawnside crossings are displayed in the left panels of Fig. 7 and 8, the right panels display the duskside crossings. Since in this section we focus on the IBL and on the interface between the IBL and magnetosphere proper, we now plot the parameters as function of a normalized time, $\breve{t}$, which is defined such that $\breve{t}=0$ corresponds to the interface between OBL and IBL and $\breve{t}=1$ corresponds to the inner edge of the boundary layer. With this choice of the time axis the magnetopause corresponds for each event to a different position $\breve{t}<0$ and changes of the parameters occurring at the magnetopause are washed out. The averaging is performed in the same manner as in Sect. 5.1, and the data are plotted for $-1<\breve{t}<2$.

For the diagrams of $V_{p L}$, and $V_{p M}$ in Fig. 4, the sign of $V_{p L}$ is again reversed for crossings at southern latitudes, and the sign of $V_{p M}$ is again reversed for crossings duskward of local noon, i.e. for the crossings plotted in the right panels of Fig. 4. In the magnetosphere proper, the average of the north-south component, $V_{p L}$, is not significantly different from zero, and it is also not significantly different from zero in the IBL on the duskside. $V_{p L}$ is directed poleward away from the equatorial plane in the IBL on the dawnside. The dawn-dusk component, $V_{p M}$, exhibits a strong change at the interface between the OBL and the IBL on the duskside. While the flow in the OBL and magnetosheath is directed toward the (dusk) flank, the plasma flow in the IBL and magnetosphere is directed sunward toward the subsolar point. However, on the dawnside, the component $V_{p M}$ of the plasma flow is directed toward the (dawn) flank in all four plasma regions. We do not know the reason for this dawndusk asymmetry of $V_{p M}$. Possibly, the evening side bulge of the plasmasphere plays a role.

The first panel of Fig. 7 shows again that the interface between the OBL and the IBL is the location where most of the density decrease from the magnetosheath level to the magnetospheric level of about $1 \mathrm{~cm}^{-3}$ takes place. At the inner edge of the boundary layer, $N$ changes by a factor of 2 on the dawnside and there is no significant change on the duskside. The last two panels of Fig. 7 show again the drop in $N_{2 p}$ and $N_{2 e}$ from the IBL to the OBL. There is also a reduction of $N_{2 e}$ from the magnetosphere proper to the IBL. On the dawnside, $N_{2 e}$ is almost one order of magnitude higher than on the duskside, and the reduction from the magnetosphere to the IBL is more pronounced.

The average proton temperature displayed in Fig. 5 increases at the interface between the OBL and the IBL and again at the inner edge of the boundary layer both for the dawnside and duskside crossings. The thermal energy of electrons, $K T_{e}$, on the dawnside increases continuously from about $60 \mathrm{eV}$ sheathward of the IBL to $300 \mathrm{eV}$ in the magnetosphere proper. On the duskside, the average electron temperature in the IBL is comparable to its value on the dawnside. However, the electron temperature in the magnetosphere proper on the duskside is considerably less than on the dawnside. We will return to this dawn-dusk asymmetry of $T_{e}$ shortly.

The proton temperature anisotropy, $T_{p \|} / T_{p \perp}$, does not vary significantly in the plotted interval $-1<\breve{t}<2$. The electron temperature is roughly isotropic in the magnetosphere, while it exhibits a strong field-aligned anisotropy, $T_{e \|} / T_{e \perp} \approx 1.2$ in the boundary layer. 


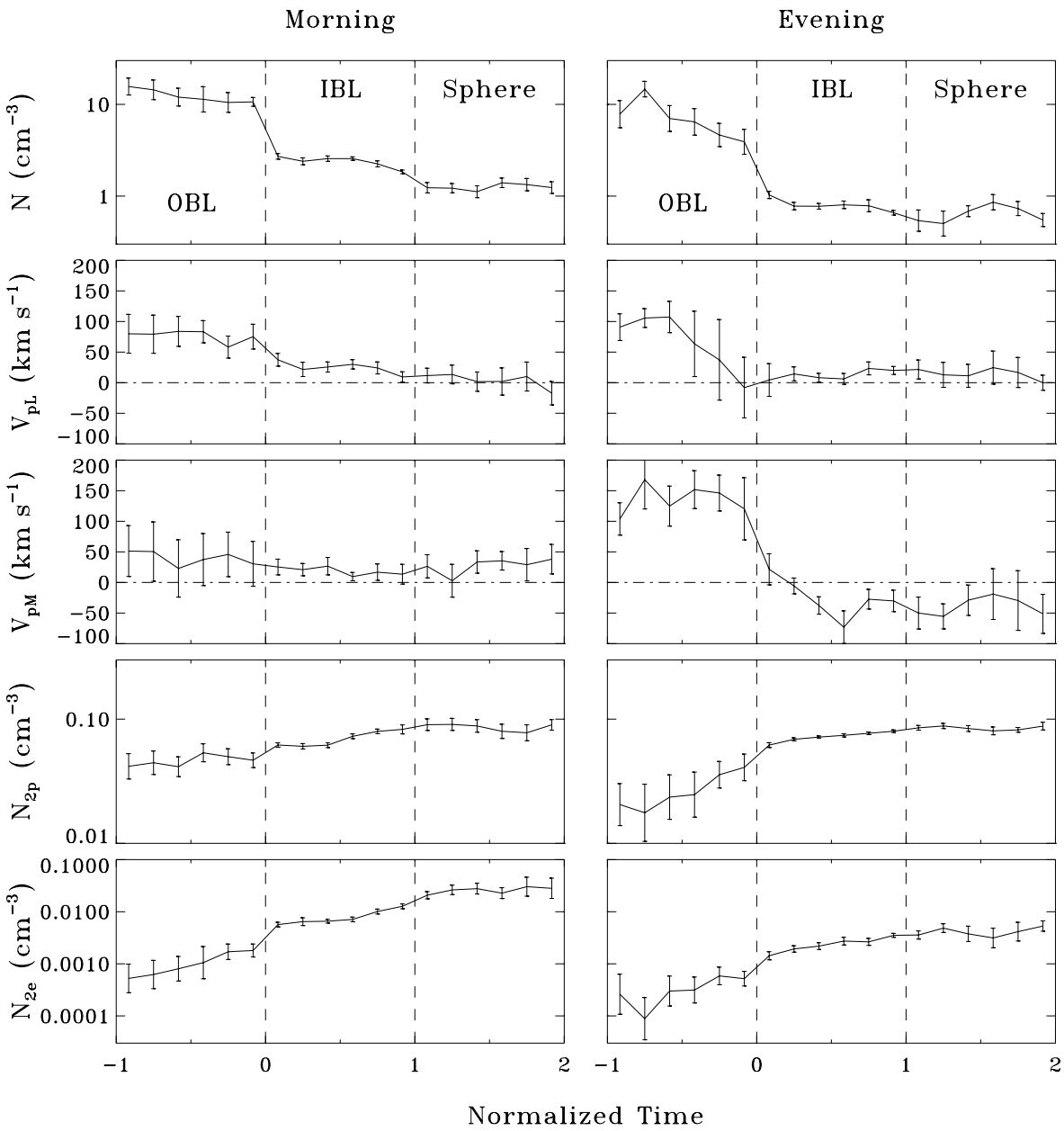

Fig. 7. Superposed epoch analysis of the components $V_{p L}, V_{p M}$ of the proton bulk velocity the total plasma density, $N$, the partial density, $N_{2 p}$, of protons above $8 \mathrm{keV}$, and the partial density, $N_{2 e}$, of electrons above $1.8 \mathrm{keV}$ across the inner boundary layer. The sign of $V_{p L}$ is reversed for crossings at southern latitudes and the sign of $V_{p M}$ is reversed for crossings duskward of local noon. The left panels display data from 13 crossings dawnward of local noon and the right panels display data from 9 crossings duskward of local noon. Normalized time $\breve{t}=0$ corresponds to the interface between the OBL and the IBL and normalized time $\breve{t}=1$ corresponds to the inner edge of the boundary layer. Vertical error bars indicate the standard deviation of the average of each parameter.
Densities at the full time resolution of one IRM spin period are available in 3 energy bands; $N_{0 p}, N_{1 p}, N_{2 p}$ are the partial densities of protons in the energy bands $20 \mathrm{eV}-$ $400 \mathrm{eV}, 400 \mathrm{eV}-8 \mathrm{keV}$, and $8 \mathrm{keV}-40 \mathrm{keV}$, respectively. $N_{0 e}$, $N_{1 e}, N_{2 e}$ are the partial densities of electrons in the energy bands $15 \mathrm{eV}-60 \mathrm{eV}, 60 \mathrm{eV}-1.8 \mathrm{keV}$, and $1.8 \mathrm{keV}-30 \mathrm{keV}$, respectively, while $N_{2 p}$ and $N_{2 e}$ are plotted in Fig. 4. In the last two panels of Fig. 8, $N_{0 p} / N_{p}$ and $N_{0 e} / N_{e}$ are given, i.e. the fraction of protons below $400 \mathrm{eV}$ and the fraction of electrons below $60 \mathrm{eV}$, respectively. $N_{0 p} / N_{p}$ is roughly constant in the interval plotted. Note, however, that the particles contributing to $N_{0 p}$ belong to different populations. While in the magnetosheath and the OBL, $N_{0 p}$ is dominated by the low energy portion of the solar wind population, it measures predominantly cold ionospheric ions in the magnetosphere. where $1-N_{0 p} / N_{p}$ and $N_{0 p} / N_{p}$ can be considered as rough estimates of the respective contributions of hot ring current ions and cold ionospheric ions to the total density. We obtain the result that there are about as many ring current ions as cold ions both in the dawnside and duskside magnetosphere. Note, however, that there may be a large number of cold ions that are not detected if their energy is below $20 \mathrm{eV}$.

In the sixth panel of Fig. 8, we recognize an increase in
$N_{0 e} / N_{e}$ at the interface between the IBL and the magnetosphere proper. This increase reflects a strong decrease in $N_{1 e}$ and is more pronounced on the duskside. The reason for the decrease in $N_{1 e}$ is the disappearance of solar wind electrons at the inner edge of the boundary layer. Siminar for ions, the respective contributions of hot ring current electrons and cold ionospheric electrons to the total density in the magnetosphere can be estimated with $1-N_{0 e} / N_{e}$ and $N_{0 e} / N_{e}$. We find that $\sim 40 \%$ of the electrons on the dawnside and $\sim 20 \%$ of the electrons on the duskside are ring current electrons. Since the IRM plasma instrument does not detect electrons below $15 \mathrm{eV}$, the actual percentage of ring current electrons is likely to be lower. The low percentage of ring current electrons on the duskside explains why the thermal energy, $K T_{e}$, of electrons measured in the magnetosphere is only $80 \mathrm{eV}$. The higher number of ring current electrons on the dawnside may be explained with the drift paths of energetic particles in the magnetosphere: when electrons (ions) energized in the magnetotail are convected sunward, they are deflected to the dawnside (duskside) due to curvature drift and gradient $B$ drift. A dawn-dusk asymmetry in the reverse sense for ring current ions has indeed been observed by Fujimoto et al. (1998b). Our traces of $N_{2 p}$ do plotted, however, not exhibit 


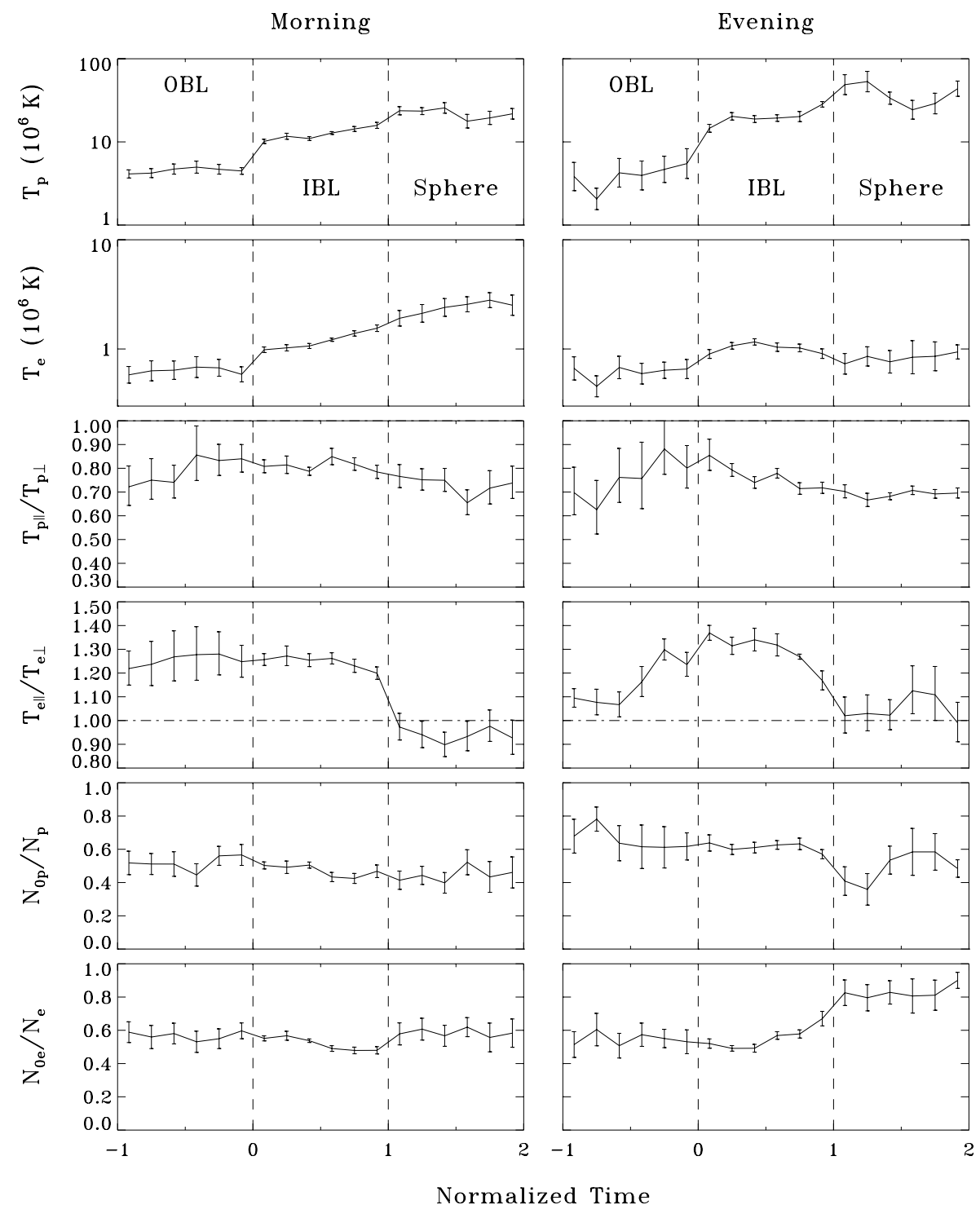

Fig. 8. Superposed epoch analysis of the proton temperature, $T_{p}$, the electron temperature, $T_{e}$, the proton temperature anisotropy, $T_{p \|} / T_{p \perp}$, the electron temperature anisotropy, $T_{e \|} / T_{e \perp}$, the fraction, $N_{0 p} / N_{p}$, of protons below $400 \mathrm{eV}$, and the fraction, $N_{0 e} / N_{e}$, of electrons below $60 \mathrm{eV}$ across the inner boundary layer. The format is the same as in Fig. 7. a dawn-dusk asymmetry for the ions.

\section{Average wave spectra and estimated diffusion}

In the present section, we evaluate average spectra of electric and magnetic fluctuations in the vicinity of the magnetopause. The electric power spectral density is directly measured by the IRM wave experiment in 16 frequency channel, and the magnetic power spectra are obtained by Fourier analysis of the high resolution magnetometer data.

\subsection{Electric fluctuations}

For each of the 16 frequency channels, the ELF/VLF spectrum analyzer provides mean values, $S_{E}$, and peak values, $\hat{S}_{E}$, of the electric power spectral density with a time resolution of $1 \mathrm{~s}$. In Fig. 9, $S_{E}$ and $\hat{S}_{E}$ are binned with respect to the boundary layer and averaged over the magnetopause crossings of the statistical data set. Each diagram of Fig. 9 corresponds to one bin. The first diagram (Sheath) includes all spectra obtained in a time interval that starts $\Delta t_{\mathrm{OBL}}$ sheathward of the magnetopause and ends $0.5 \Delta t_{\mathrm{OBL}}$ sheathward of the magnetopause. The second diagram (Sheath/MP) includes all spectra obtained in a time interval that starts $0.5 \Delta t_{\mathrm{OBL}}$ sheathward of the magnetopause and ends at the magnetopause. The next four diagrams (OBL/MP, OBL, IBL, IBL/IE) include all spectra obtained in the sheathward half of the OBL, in the earthward half of the OBL, in the sheathward half of the IBL, and in the earthward half of the IBL, respectively. In the last diagram, all spectra are included that are obtained in the magnetosphere less than $0.5 \Delta t_{\mathrm{IBL}}$ earthward of the interface between the IBL and the magnetosphere. The spectra plotted on the left side of Fig. 9 are based on all crossings of the data set that have an OBL, and the spectra plotted on the right side are based on all crossings that have an IBL. 




Fig. 9. Spectra of electric fluctuations in the vicinity of the boundary layer averaged over the crossings of the data set. Each diagram displays mean values, $S_{E}$ (lower curve), and peak values, $\hat{S}_{E}$ (upper curve), of the electric power spectral density in a normalized time interval. $S_{E}$ is averaged within the interval of one event and then again averaged over all events. For $\hat{S}_{E}$ the maximum within the interval is taken for each event and then the maxima are averaged over all events. On the left side, we see from top to bottom average spectra in the magnetosheath $(-1<\hat{t}<-0.5)$, in the magnetosheath close to the magnetopause $(-0.5<\hat{t}<0)$, in the sheathward half of the OBL $(0<\hat{t}<0.5)$, and in the earthward half of the OBL $(0.5<\hat{t}<1)$. On the right side, we see from top to bottom average spectra in the sheathward half of the IBL $(0<\breve{t}<0.5)$, in the earthward half of the IBL $(0.5<\breve{t}<1)$, and in the magnetosphere $(1<\breve{t}<1.5)$. Vertical error bars indicate the standard deviation of the average of $S_{E}$ and $\hat{S}_{E}$. The dash-dotted line gives the noise level. Crosses and diamonds with horizontal error bars give the average and standard deviation $( \pm \sigma)$ of the electron gyrofrequency and electron plasma frequency, respectively.
The mean values, $S_{E}$, of one event are averaged within one bin and then again averaged over all events. For the peak values, $\hat{S}_{E}$, we take the maximum within one bin for each event and then average the maxima over all events. In each diagram of Fig. 9, the peak values are much higher than the mean values, which indicates that the power of the fluctuations is very variable.

Below the electron gyrofrequency, $f_{g e}$, all spectra of the mean power, $S_{E}$, in Fig. 9 follow a power law, $S_{E} \propto f^{-\epsilon}$, with $2.1<\epsilon<2.5$. In the magnetosphere, electric fluctuations in that frequency range are known to be due to whistler mode chorus (Tsurutani and Smith, 1977) excited by trapped ring current electrons. The power law spectrum below $f_{g e}$ in the magnetosheath and boundary layer was reported by
Gurnett et al. (1979). They concluded that these electric fluctuations are partly due to whistler waves and to electrostatic emissions that are referred to as broad-band electrostatic noise. The lower hybrid drift instability and the electrostatic ion cyclotron instability have been invoked as possible sources of the broad-band electrostatic noise.

Figure 9 shows that the wave power below $800 \mathrm{~Hz}$ maximizes, on average, in the earthward half of the OBL and sheathward half of the IBL. This finding can be highligned in Figure 10 by plotting the superposed epoch traces of the root-mean-square amplitudes, $\delta E_{1}$ and $\delta \hat{E}_{1}$, of channels 1-6 $(25-800 \mathrm{~Hz}) . \delta E_{1}$ is computed by integrating channels $1-6$, after $S_{E}$ has been averaged within one bin for each event. $\delta \hat{E}_{1}$ is computed by integrating channels $1-6$, after the maxi- 


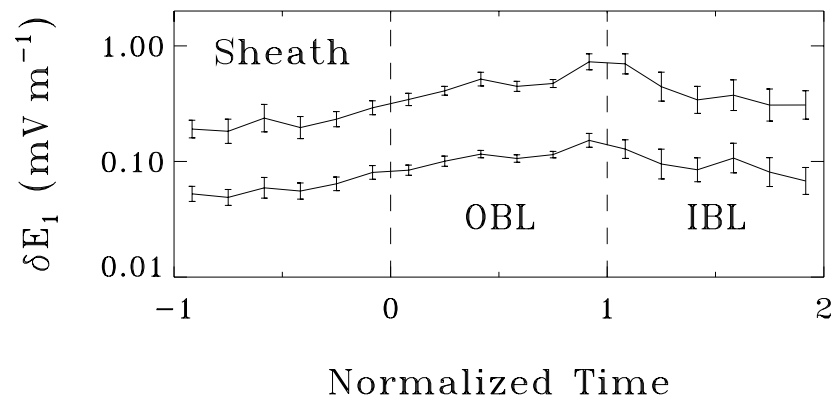

Fig. 10. Superposed epoch analysis of the root-mean-square amplitudes of electric fluctuations in the range of $25-800 \mathrm{~Hz}, \delta E_{1}$ and $\delta \hat{E}_{1}$, across the outer boundary layer.

mum of $\hat{S}_{E}$ within one bin has been taken for each event. $\delta E_{1}$ and $\delta \hat{E}_{1}$ are enhanced at the interface between the OBL and the IBL, which is the location of the strongest density gradient (Figs. 2 and 4). The connection between the maximum of $\delta E_{1}$ and the strongest density gradient argues for the effect of the transverse density gradient as required by the lower hybrid drift instability. On the other hand, Sazhin et al. (1991) suggested that enhanced wave power at the location of the strongest density gradient may be caused by reflection or the trapping of whistler waves. Lower hybrid waves near the resonance cone propagate on the same branch as whistlers and may transform into whistler waves and vice versa.

At about half the electron gyrofrequency, the average spectra of the mean power, $S_{E}$, in the magnetosphere and IBL start to deviate from the power law observed at low frequencies. Between the electron gyrofrequency, $f_{g e}$, and the electron plasma frequency, $f_{p e}$, they are pretty flat, before they are finally cut off above $f_{p e}$. The electric fluctuations measured above $f_{g e}$ are due to electrostatic electron cyclotron emissions and upper hybrid waves (Anderson et al., 1982), which are excited by trapped ring current electrons.

In the magnetosheath and OBL, the average spectra of $S_{E}$ show a similar deviation from the power law near $f_{g e}$ and a bump at the plasma frequency. The deviation near $f_{g e}$ is probably also caused by electrostatic electron cyclotron emissions. Moreover, electron acoustic waves may contribute to the power in that frequency range (LaBelle et al., 1987). The bump at $f_{p e}$ is due to Langmuir waves or upper hybrid waves. Comparing the observed power at $f_{p e}$ with the thermal fluctuation level (e.g. Baumjohann and Treumann, 1996) of Langmuir waves in an unmagnetized Maxwellian electron plasma with thermal energy, $K T_{e} \approx 50 \mathrm{eV}$, which is typical for the magnetosheath, we find that the mean power is comparable to that thermal level, whereas the peak power is 2 orders of magnitude higher and must be caused by a nonthermal source. Gurnett et al. (1979) reported that the emissions at the plasma frequency occur in bursts, which explains the large difference between $\hat{S}_{E}$ and $S_{E}$.

According to Thorne and Tsurutani (1991), the average power spectra of electric and magnetic fluctuations measured in the boundary layer close to the magnetopause on board the
ISEE satellites have been approximated by the expressions

$$
\begin{aligned}
& S_{E}=\left(\frac{f}{1 \mathrm{~Hz}}\right)^{-2.8} \cdot 30 \frac{\mathrm{mV}^{2}}{\mathrm{~m}^{2} \mathrm{~Hz}} \\
& S_{B}=\left(\frac{f}{1 \mathrm{~Hz}}\right)^{-3.9} \cdot 10 \frac{\mathrm{nT}^{2}}{\mathrm{~Hz}}
\end{aligned}
$$

for frequencies $f>10 \mathrm{~Hz}$. At $1 \mathrm{kHz}$, the average power observed by IRM in the OBL and IBL is comparable to expression (3). However, since the spectral slope that we find for the broad-band electrostatic noise $(2.1<\epsilon<2.5)$ is less than the value of 2.8 given by Thorne and Tsurutani (1991), our average spectra are almost one order of magnitude lower at $30 \mathrm{~Hz}$. This difference is presently not understood, since the instruments on ISEE and IRM are similar and differ only in antenna length. Expression (4) can be compared with power spectra of the high resolution magnetometer data (next subsection). These are available up to the Nyquist frequency of $16 \mathrm{~Hz}$. We find that the magnetic power obtained at about $10 \mathrm{~Hz}$ is consistent with expression (4).

\subsection{Magnetic fluctuations}

Magnetic field readings of AMPTE-IRM are usually taken with a sampling rate of $32 \mathrm{~Hz}$. For the spectral analysis, we multiply the time series of the components of $\boldsymbol{B}$ with a $4 \mathrm{~min}$ wide Hanning window. Since the Hanning window tapers at its edges, we overlap subsequent windows and compute the power spectra every $2 \mathrm{~min}$. Thereby, the field vectors are decomposed into (1) a component along the average magnetic field, $\langle\boldsymbol{B}\rangle$, of the respective 2 min interval, (2) a component along $(\boldsymbol{n} \times\langle\boldsymbol{B}\rangle) \times\langle\boldsymbol{B}\rangle$, and (3) a component along $\boldsymbol{n} \times\langle\boldsymbol{B}\rangle$, where $\boldsymbol{n}$ is the magnetopause normal taken from the model of Fairfield (1971). Thus, we obtain spectra of compressional fluctuations, $S_{B_{\|}}$, transverse fluctuations normal to the magnetopause, $S_{B_{n \perp}}$, and transverse fluctuations tangential to the magnetopause, $S_{B_{t \perp}}$. Prior to Fourier transformation, the linear trend of the magnetic field components is removed. As described by Bauer et al. (1995), the spacecraft noise at the spin harmonics is finally eliminated.

In Figure 11, the computed power spectra are binned with respect to the boundary layer and averaged over the magnetopause crossings of the statistical data set. The averaging is done in the same way as for the mean power $S_{E}$ in the last subsection. The bin into which a given spectrum is sorted is determined by the center time of the corresponding $2 \mathrm{~min}$ interval. A spectrum is sorted out if IRM does not stay in the same region during the 2 min interval.

For frequencies $f<0.5 f_{g p}$, the average spectra in the magnetosheath follow power laws, $S \propto f^{-\epsilon}$, with spectral slopes $1.2<\epsilon<1.6$ considerably flatter than the electric spectra discussed in the previous section. The average spectra of magnetic fluctuations normal to the magnetopause, $S_{B_{n \perp}}$, are flatter than $S_{B_{\|}}$and $S_{B_{t \perp}}$. Above the proton gyrofrequency the average spectra in the magnetosheath become distinctly steeper than $f^{-2}$. The change in the slope at $f \approx 0.5 f_{g p}$ may be due to the presence of electromagnetic 
Table 2. Diffusion coefficients for solar wind particles in the OBL and IBL. The upper four rows give averages for the magnetic field magnitude, $B$, the density, $N$, and the field-aligned and perpendicular thermal energies, $K T_{s \|}$ and $K T_{s \perp}$, of solar wind protons $(s=p)$ and electrons $(s=e)$. These quantities are combined with typical amplitudes of electric and magnetic fluctuations in order to estimate proton and electron diffusion coefficients caused by lower hybrid drift instability $\left(D_{\mathrm{LH}}\right)$, gyroresonant pitch-angle scattering due to electrostatic $\left(D_{s, \mathrm{ES}}\right)$ and electromagnetic $\left(D_{s, \mathrm{EM}}\right)$ waves, and by kinetic Alfvén wave turbulence $\left(D_{s, \mathrm{KA}}\right)$. The relevant wave amplitudes are given one row above the respective diffusion coefficients

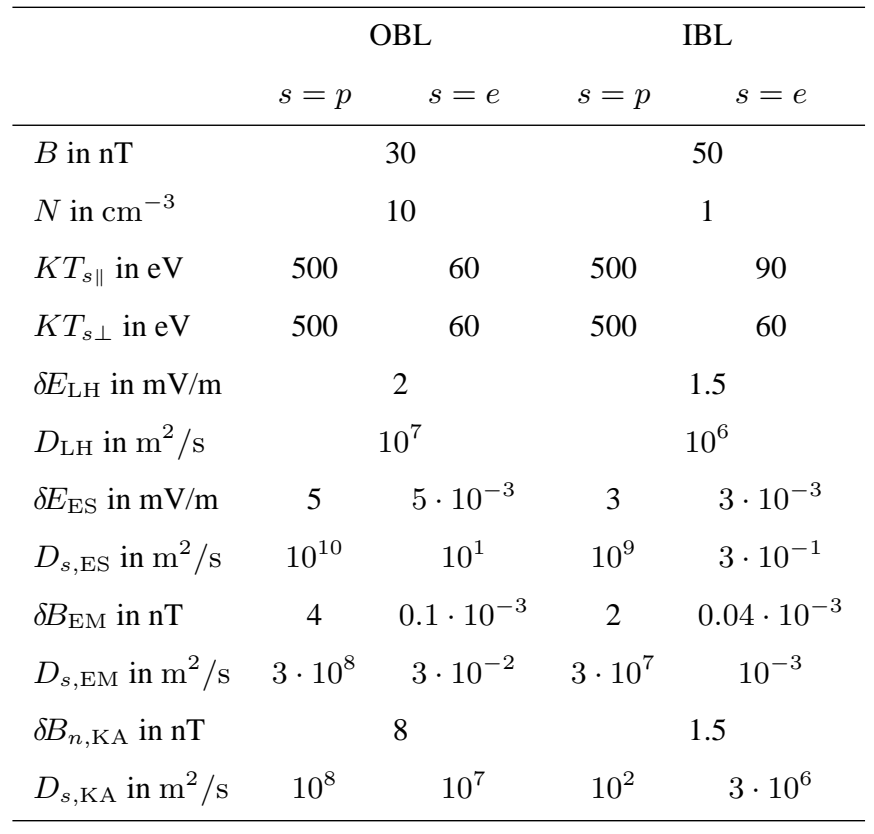

ion cyclotron waves, which were reported by, for example, Anderson et al. (1991). Electromagnetic ion cyclotron emissions can also be seen in the magnetosphere (e.g. Treumann et al., 1995), but do not show up in the average spectra of Fig. 11. The dominance of the compressional component at the lowest frequencies in the magnetosheath is due to mirror waves and the low-frequency part of magnetosonic waves (e.g. Denton et al., 1995).

Progressing from the magnetosheath to the magnetosphere, we recognize that the power below $0.3 \mathrm{~Hz}$ in the three components of $\boldsymbol{B}$ stays about constant until the outer half of the OBL and then decreases continuously. It is reasonable to assume that magnetic fluctuations below $0.3 \mathrm{~Hz}$ in the boundary layer are driven by the observed turbulence in the magnetosheath. These waves may, therefore, penetrate the boundary layer up to about half the thickness of the OBL, where they are either absorbed or transformed into other waves. For frequencies above $1 \mathrm{~Hz}$, however, the magnetic power maximizes at the magnetopause. This is consistent with ISEE measurements (e.g. Thorne and Tsurutani, 1991).

The maximum of the magnetic fluctuation at this place may have a number of causes. Either the magnetopause is capable of locally generating magnetic fluctuations in the frequency range of $f>1 \mathrm{~Hz}$ or the increase in the higher frequency wave power results from wave transformation. In this respect, it is interesting to note that a number of such transformation mechanisms have been discussed in the past referring to impinging sheath wave resonance in the magnetopause boundary layer (e.g. Southwood, 1974; Belmont et al., 1995; Zhu and Kivelson, 1988, 1989) and have been summarized in Rezeau and Belmont (2001). Very recently, however, Belmont and Rezeau (2001) have shown that it is probable that the transformation of sheath waves hitting the magnetopause is of a non-resonant nature and can be amplified over a broad range of wavelengths corresponding to a broad range of frequencies. This is due to the fact that those waves experience the density gradient in the magnetopause with the Hall and finite Larmor radius effects playing a fundamental role in the transformation process. Interestingly, Belmont and Rezeau (2001) find that the amplified waves which accumulate in the transition region have shorter wavelengths. This would explain the increase in frequency coinciding with the decrease in the low frequency wave power observed here. Belmont and Rezeau (2001) reach, however, unrealistically high amplitudes. This effect is attributed by them to their neglect of finite Larmor radius effects. Indeed, inclusion of such effects introduces a kind of damping of the waves by detuning and dissipation. Johnson and Cheng (1997) have investigated the interaction of sheath waves with the magnetopause, including finite Larmor radius corrections.

\subsection{Estimates of diffusion coefficients}

Deconvolving the time series with the measured proton bulk velocity, $V_{p n}$, normal to the magnetopause, Phan and Paschmann (1996) performed a statistical survey of the thickness of the dayside low-latitude boundary layer. They inferred an average thickness (geometric average) of $\sim$ $1000 \mathrm{~km}$, both for high and low magnetic shear. The inner edge of the boundary layer was defined as the point where the total (proton) density, $N_{p}$, drops below $5 \%$ of its magnetosheath value. Since the sharpest drop of the density is observed at the interface between the OBL and the IBL, the thickness derived by Phan and Paschmann (1996) might be taken as the thickness of the OBL rather than the thickness of the entire boundary layer. Combining this result with our finding that the average durations of the OBL and IBL are comparable (Sect. 3), we may conclude that both sublayers are on average roughly, $1000 \mathrm{~km}$ thick.

We have presented evidence that the IBL us large-scale on closed field lines. Thus, the question arises whether the solar wind particles observed in the IBL enter by means of crossfield diffusion from the OBL. Diffusion in the boundary layer is an ongoing subject of controversy, for more recent reviews of some viewpoints see, (e.g. Treumann and Skopke, 1999). The problem has not been settled yet and depends heavily on the view of (1) how diffusive processes are defined, and (2) how they can be observed. Diffusion makes sense only in an 


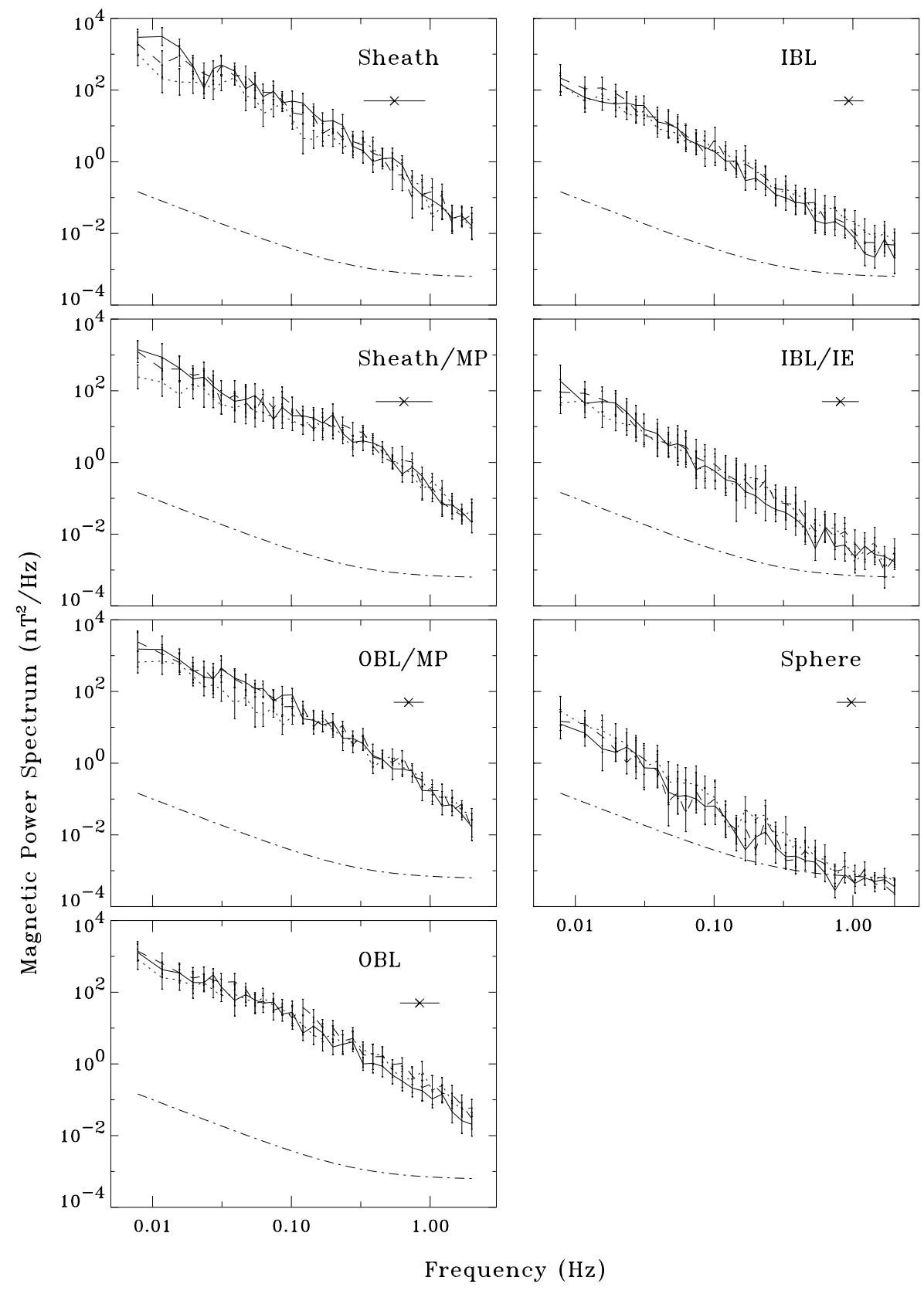

Fig. 11. Spectra of magnetic fluctuations in the vicinity of the boundary layer averaged over the crossings of the data set. Each diagram displays power spectra of compressional fluctuations, $S_{B_{\|}}$(solid line), transverse fluctuations normal to the magnetopause, $S_{B_{n \perp}}$ (dotted line), and transverse fluctuations tangential to the magnetopause, $S_{B_{t \perp}}$ (dashed line), in a normalized time interval. The spectra are averaged within the interval of one event and then again averaged over all events. On the left side, we see from top to bottom spectra in the magnetosheath $(-1<$ $\hat{t}<-0.5)$, in the magnetosheath close to the magnetopause $(-0.5<\hat{t}<0)$, in the sheathward half of the OBL $(0<$ $\hat{t}<0.5)$, and in the earthward half of the OBL $(0.5<\hat{t}<1)$. On the right side, we see from top to bottom spectra in the sheathward half of the IBL $(0<\breve{t}<0.5)$, in the earthward half of the IBL $(0.5<\breve{t}<1)$, and in the magnetosphere $(1<\breve{t}<1.5)$. Vertical error bars indicate the standard deviation of the average of $S_{B_{\|}}, S_{B_{n \perp}}$, and $S_{B_{t \perp}}$. The dash-dotted line gives the noise level. Crosses with horizontal error bars give the average and standard deviation $( \pm \sigma)$ of the proton gyrofrequency. inhomogeneous plasma. It will thus be confined to regions of gradients in any of the plasma and field quantities (density, pressure, flow, and magnetic field). Moreover, one can take the microscopic or the macroscopic viewpoint. Microscopically, one needs to specify the very mechanism of local particle (or field) scattering, while macroscopically, one assumes given transport coefficients and considers large-scale turbulent transport. Both views have been taken in the literature with more or less diverging results. Clearly, the microscopic approach is the more consistent one; it has, however, in many cases to be combined with the macro-viewpoint in order to do justice to reality. On the other hand, the microscopic view requires precise knowledge of the relevant micro-instabilities, which in most cases, is not available. Micro-instabilities are usually treated only in velocity space. When accounting for the inclusion of configuration space inhomogeneities, various effects like particle drift, finite Larmor radius effects and wave transformation have to be taken into account. This can have a strong influence on the evolution of the microinstabilities and thus modify their importance. Recently, Rezeau and Belmont (2001) and Belmont and Rezeau (2001) have discussed the effect of inhomogeneities on the evolution of low frequency waves when interacting with the magnetopause and have shown that non-locally generated waves convecting in from the magnetosheath can have strong effects on transport in the magnetopause layer. We will briefly come to this point below. Here, at the expense of a discussion of the latter, we take for the moment the micro-viewpoint. We use the 
available spectral information about the plasma turbulence in order to estimate the micro-diffusion coefficient. Interpreting the spectra in terms of the well developed nonlinear state of some micro-instabilities, one estimates the diffusivities. We will then briefly discuss the relevance of this approach.

Let us first focus on the differences between diffusion in the outer and inner boundary layer. If the OBL is formed by inward diffusion, then its thickness, $h_{\mathrm{OBL}}$, at a certain location is given by the diffusion coefficient, $D_{\mathrm{OBL}}$, and the time $\tau_{\mathrm{OBL}}$ that has passed since the flux tubes first came into contact with the magnetopauseyields $h_{\mathrm{OBL}} \sim\left(D_{\mathrm{OBL}} \tau_{\mathrm{OBL}}\right)^{1 / 2}$. At the dayside magnetopause the contact time $\tau_{\mathrm{OBL}}$ is of the order of $10 \mathrm{~min}$, corresponding to a distance of $15 R_{E}$ traveled at a flow speed of $150 \mathrm{~km} / \mathrm{s}$ which is typical for the OBL. Combining this with a typical thickness, $h_{\mathrm{OBL}} \sim 1000 \mathrm{~km}$, of the OBL, one arrives at the often quoted result (Skopke et al., 1981) that $D_{\mathrm{OBL}} \sim 10^{9} \mathrm{~m}^{2} / \mathrm{s}$ is needed to populate the OBL by means of cross-field diffusion. For the diffusion of solar wind particles from the OBL into the IBL, the time $\tau_{\text {IBL }}$ that has passed since the flux tube in the IBL first came into contact with the interface between the sublayers is relevant. Since the flow in the IBL is distinctly slower than in the OBL and often directed sunward rather than tailward, $\tau_{\text {IBL }}$ may be considerably longer than $\tau_{\mathrm{OBL}}$.

Table 2 lists the estimates for the diffusivities in the OBL and IBL caused by the lower hybrid drift instability, gyroresonant pitch angle scattering, and kinetic Alfvén wave turbulence, respectively. The lower hybrid drift instability may be driven by the cross-field (density and temperature gradientdrift, shear flow) current in the magnetopause (Treumann et al., 1992a, 1995; Winske et al., 1995; Winske and Omidi, 1995). The quasilinear anomalous resistivity associated with this instability should lead to ambipolar diffusion with a common diffusion coefficient for protons and electrons given by

$D_{\mathrm{LH}} \approx 0.6 r_{g e}^{2} \omega_{\mathrm{LH}} \frac{m_{p} \omega_{p e}^{2}}{m_{e} \omega_{g e}^{2}}\left(1+\frac{T_{p \perp}}{T_{e \perp}}\right) \frac{\epsilon_{0} \delta E^{2}}{2 N K T_{p \perp}}$

where $\omega_{p s}$ and $\omega_{g s}$ are the plasma frequency and gyrofrequency of species $s$ ( $s=p$ for protons, $s=e$ for electrons). The thermal speed and gyroradius of species $s$ are defined as $v_{T s}=\left(K T_{s} / m_{s}\right)^{1 / 2}$ and $r_{g s}=v_{T s} / \omega_{g s} . \omega_{\mathrm{LH}}$ is the lower hybrid frequency and $\delta E$ is the electric field amplitude of the waves. For the estimate of $D_{\mathrm{LH}}$ in Table 2, we assume that the lower hybrid drift instability is responsible for the electric power observed in the frequency range $0.1-10 f_{\mathrm{LH}}$ and compute $\delta E$ by integrating Eq. (3) over that frequency range. Since the anomalous resistivity associated with other cross-field current-driven instabilities are much less than the lower hybrid drift resistivity (Winske et al., 1995; Treumann et al., 1992a, 1995), diffusion coefficients based on those instabilities are considerably lower than the one given in Eq. (5). The philosophy of such an approach to anomalous diffusion has been widely discussed in the literature, starting from an investigation of the neo-classical banana regime which was fashionable in the 1960s. The idea is that current or gradient driven electrostatic waves scatter the particles by their electric wave fields, similar to particle collisions. The wave spectrum is generated by non-linear cascading into sidebands. This process cannot be resolved in a simple way by measurements, such as the ones we are basing our discussion on. Therefore, we are forced to restrict the application to taking the wave spectrum for granted as the final result of the cascading process (e.g. Sagdeev, 1979). Nonlinear evolution of the diffusivity is a complicated process. It has been phenomenologically treated in work on plasma turbulence (Sagdeev, 1979) and on Lévy flights (Treumann, 1997). In the nonlinear regime, the diffusivity becomes timedependent. The latter reference has demonstrated that it is usually less than any quasilinear estimate which identifies the estimates given in Table 2 as upper limits. In any case, they are only crude estimates in light of the assumptions, approximations and neglects discussed below.

When abandoning microscopic anomalous collisions, one may assume that pitch-angle scattering causes increases in the gyroradii until the particles move to a new magnetic flux tube. This approach is equivalent to postulating that the collision frequency of the particles equals the coefficient of pitch angle diffusion by gyroresonant waves. Thorne and Tsurutani (1991) derive the expressions for the spatial proton $(s=p)$ and electron $(s=e)$ diffusion coefficients

$$
\begin{aligned}
& D_{s, \mathrm{ES}} \sim r_{g s}^{2} \omega_{g s}\left(\frac{\delta E}{v_{T s} B}\right)^{2} \\
& D_{s, \mathrm{EM}} \sim r_{g s}^{2} \omega_{g s}\left(\frac{\delta B}{B}\right)^{2}
\end{aligned}
$$

in this case associated with pitch angle scattering (PAS) by electrostatic (ES) and electromagnetic (EM) waves, respectively. In Table 2 , we estimate $\delta E$ and $\delta B$ by integrating Eqs. (3) and (4) from $0.9 f_{g s}$ to $1.1 f_{g s}$. As for a general criticism of this method, we note that this kind of gyro-resonance affects only resonant particles which occupy only a narrow range of the particle phase space (essentially of measure zero). Therefore, the main application of this kind of diffusion is to scatter sufficiently energetic particles in a plasma, such as cosmic rays crossing the interplanetary space. Nonresonant and, in particular, low-energy particles, such as the solar wind-magnetosheath plasma, remain immune against pitch-angle scattering.

Kinetic Alfvén waves in the boundary layer may be generated due to the coupling of the turbulence in the magnetosheath (Lee et al., 1994). Resonant amplification (Belmont et al., 1995) of magnetosheath waves at the magnetopause gradient may also be a cause of such waves appearing in the magnetopause, though it has recently been argued (Belmont and Rezeau, 2001) that the resonant amplification plays a considerably minor role here. These waves will certainly evolve nonlinearly by various processes, even forming solitary structures (Rezeau et al., 1993) and causing field-line resonances (Lotko and Sonnerup, 1995; Streltsov and Lotko, 
1996). They will lead to anomalous diffusion as well (Lotko and Sonnerup, 1995). The associated diffusion coefficient

$D_{s, \mathrm{KA}} \approx \frac{0.6 c_{A}^{2}}{k_{\|} v_{T s \|}} \exp \left(-\frac{c_{A}^{2}}{2 v_{T s \|}^{2}}\right)\left(\frac{T_{e \perp}}{T_{p \perp}} k_{\perp}^{2} r_{g p}^{2} \frac{\delta B_{n}}{B}\right)^{2}$

depends on the amplitude $\delta B_{n}$ of magnetic fluctuations normal to the magnetopause and on the components $k_{\perp}$ and $k_{\|}$ of the wave vector $\boldsymbol{k}$ perpendicular and parallel to $\boldsymbol{B}$. For our estimate of $D_{s, \mathrm{KA}}$ in Table 2, we assume $k_{\perp} r_{g p}=0.5$ and $\lambda_{\|}=2 \pi / k_{\|}=5 R_{E}$. The amplitude $\delta B_{n}$ is computed by integrating the average power $S_{B_{n \perp}}$ of Fig. 11 from $0.5 c_{A} / \lambda_{\|}$ to $2 c_{A} / \lambda_{\|}$. The estimate of $D_{s, \mathrm{KA}}$ is thus based on the assumption that the full power $S_{B_{n \perp}}$ observed in that frequency range is due to kinetic Alfvén waves.

Table 2 suggests that cross-field diffusion by lower hybrid waves cannot explain the formation of the boundary layer. The value found for $D_{p \text {,ES }}$ in the OBL is one order of magnitude higher than required for the transport of solar wind protons into that sublayer, but pitch angle scattering by gyroresonant waves cannot explain the presence of solar wind electrons in the boundary layer. Note that an ambipolar electric field generated by the fast ion diffusion will enhance $D_{e}$ only by a factor of $1+T_{p \perp} / T_{e \perp}$ which is of importance only in extremely low electron temperature plasmas. In the magnetosheath-magnetopause region, this factor is only of the order of $\sim 8$. Hence, the electrons will hold the ions back, even when the latter would diffuse fast enough. Their motion, nevertheless, requires breaking the frozen-in condition. This must be achieved by instability involving the electrons, i.e. in order to neutralize the plasma electrons, field-aligned currents are formed which generate anomalous resistance and scatter the electrons into the direction of the ion flow. Moreover, the ions behave non-magnetized over the distance of their gyroradii, which leads to the excitation of lower-hybrid waves. Both mechanisms lead back to the above discussed current driven instabilities and thus cannot be faster than those.

In the IBL, $D_{\mathrm{LH}}, D_{s, \mathrm{ES}}$, and $D_{s, \mathrm{EM}}$ are all about one order of magnitude lower than in the OBL. Since $D_{p, \mathrm{KA}}$ is very sensitive to the ratio $c_{A} / v_{T s \|}$, its value in the IBL is 6 orders of magnitude lower than in the OBL, whereas the values of $D_{e, \mathrm{KA}}$ in the IBL and OBL are not very different. Among the diffusion coefficients considered, $D_{e, \mathrm{KA}}$ is the best candidate for explaining the entry of warm electrons from the magnetosheath onto closed field lines in the IBL. However, with $D_{\text {IBL }} \sim D_{e, \mathrm{KA}} \sim 3 \cdot 10^{6} \mathrm{~m}^{2} / \mathrm{s}$, a contact time $\tau_{\mathrm{IBL}}$, of the order of 100 hours is needed to form an IBL of the thickness $\delta_{\mathrm{IBL}} \sim 1000 \mathrm{~km}$, which is too long, even in view of the stagnant flow observed in the IBL. In summary, apparently none of the diffusion mechanisms considered can explain the presence of both solar wind protons and electrons in the OBL or IBL. This rules out purely anomalous diffusion based solely on velocity space instabilities.

We note that Treumann et al. (1992b) directly determined diffusion coefficients from the density profiles in the boundary layer and found that the diffusivities were low and roughly in agreement with microscopic theory. The apparent contradiction to numerical simulations presented, for instant, by Winske and Omidi (1995) and Winske et al. (1995) were resolved when applying the same methods to the simulation profiles. The diffusion coefficients there were consistent with microscopic theory as well, while the diffusivities determined from the first arrival of particles at a certain position were much higher, at approximately the order of the diffusivity needed for the explanation of the boundary layer by diffusion. However, the arrival of the first scattered particles at some position is not significant for a diffusive process, for diffusion is an average statistical process and applies to the bulk distribution and not to a small, insignificant number of single scattered particles, as has been argued in Treumann et al. (1992b).

Diffusion in this section has been treated on the basis of micro-processes, assuming the presence of microinstabilities. One possibility of saving a microscopic diffusion process may be based on the assumption that the wave power in the resulting spectra is concentrated in much smaller patches than can be resolved by the instrumentation. In this case, the power will be locally large and cause much stronger diffusivities locally than estimated here. Elsewhere (Treumann et al., 1995), we have argued that lower-hybrid drift waves will actually form such small structures of concentrated wave power. The process is, however, not restricted to this particular kind of waves. One knows that ion sound waves and electron acoustic waves form small-scale nonlinear patches called solitary structures of ion holes, respectively, electron holes. Particles are mixed here and may become accelerated both along and perpendicular to the magnetic field. Such effects should cause strong though localized diffusion. However, confirmation requires better resolution of the particle and field measurements.

On the other hand, coupling between micro- and macroprocesses could lead as well to an important modification of the diffusive concept (Treumann and Skopke, 1999). Such a coupling can be three-fold. First, assume that the micro- and macro-instabilities are essentially decoupled, which is the simpler case. In the presence of macroscopic eddies caused by macro-instabilities, such as the Kelvin-Helmholtz instability and its nonlinear relatives or by wave vortices transported from the magnetosheath by convection, the plasma undergoes large-scale motions that steer the magnetic flux tubes without the necessity for diffusive processes or reconnection to be generated by the large-scale turbulence. Even when the flux tubes retain their identity and do not reconnect, a small amount of micro-diffusivity along the path of a flux tube caused by micro-instabilities will be sufficient to transport plasma during the slow turbulent eddy motion from one flux tube to another neighboring flux tube. In this case, the main transport is provided on the large-scale by the macroscopic eddy motion, while micro-diffusion just cares for the exchange of plasma across the borders of flux tubes on the small scale of the inter-flux tube distances. Diffusivities in such a model need not be extraordinarily large and could well remain of the order of the estimates presented here. This 
is the simplest imaginable non-reconnective plasma transport based on very weak microscopic diffusivity and macroscopic turbulent motion.

In the second case, micro-and macroprocesses may be coupled directly in the presence of sufficiently steep gradients when both wave transformation and finite Larmor radius effects must be taken into account. Such effects have been discussed by Galeev et al. (1986) and reviewed by Rezeau and Belmont (2001). We will briefly return to them below. A third possibility is based on the idea that the large-amplitude, very low-frequency turbulence present in the magnetosheath may resonantly and nonresonantly interact with the magnetopause density/temperature/field/velocity gradient layer (Johnson and Cheng, 1997; Belmont and Rezeau, 2001) in a way that the turbulence is transformed in frequency and mode and accumulates in the transition layer, as mentioned earlier in this paper. A broad spectrum of such low-frequency turbulence will of course lead to a mixing of plasma and magnetic field flux tubes on short scales and may, therefore, contribute to microscopic and possibly even patchy reconnection. One may infer that such processes might lead to scale invariant reconnection, in which case the power law spectra observed in the waves at low frequencies will find a relatively natural explanation. It is assumed (Belmont and Rezeau, 2001) that in this case, no micro-instabilities may be required. Reconnection providing a diffusive process of the type of flux-tube diffusivity (or percolation) was originally proposed by Rosenbluth et al. (1966) and applied to magnetopause processes by Galeev et al. (1986). Based on that theory, LaBelle and Treumann (1988) had already estimated reasonably high magnetic mixing diffusivities that clearly could account for the generation of the entire OLB and ILB by magnetic flux tube diffusion in turbulent percolative micro-reconnection. Belmont and Rezeau (2001) have provided a first solution to the problem of transformation of the fundamental magnetohydrodynamic (MHD) waves at the magnetopause in Hall-magnetohydrodynamic, while still neglecting finite Larmor radius effects. These results look very promising and may provide a first clue to the treatment of turbulent reconnection driven by magnetosheath wave processes. Thus, in this case, the energy source of the turbulent transport at the magnetopause may be sought for the persistent presence of large amplitude, large-scale magnetosheath turbulence. It should, however, be mentioned that in the presence of such an intense wave spectrum and relatively steep local gradients, one expects that the macro-turbulence will readily couple to micro-turbulence, thereby increasing the diffusivity locally and additionally supporting the evolution of reconnection. The real reconnection process will thus be a mixture of a locally diffusive and on the larger scale magnetically turbulent phenomenon.

\section{Discussion}

The analysis of the AMPTE/IRM magnetopause crossings presented a variety of information on the structure of the day- side, low-latitude boundary layer. Let us now use this information to discuss how the boundary layer is formed.

\subsection{Formation of the outer boundary layer}

In Paper I, we found that for the 13 Walén events in the data set, the measurements taken in the vicinity of the magnetopause fulfill a linear relation of the type of Eq. 2. Is Eq. (2) fulfilled in the entire OBL or only in a small portion close to the magnetopause? For 2 of the 13 Walén events it is not possible to distinguish between the OBL and the IBL. For 2 Walén events, Eq. (2) is fulfilled only in a small portion (a few percent) of the OBL close to the magnetopause. One of these two events is the magnetopause crossing on $30 \mathrm{Au}-$ gust 1984 at 09:56:43. In Paper I, it was mentioned that during that crossing the counterstreaming of solar wind and cold ions is inconsistent with the sign of $B_{n}$ inferred from Eq. (2). Obviously, only a small portion of the OBL is on field lines that cross the magnetopause locally. For one Walén event Eq. (2) is fulfilled for $\sim 80 \%$ of the OBL. For the remaining 8 Walén events, Eq. (2) is fulfilled over the entire OBL, which indicates that the entire OBL is on open field lines.

In the case of the 27 non-Walén events, we do not find the correlation between $\boldsymbol{V}_{p}$ and $\boldsymbol{c}_{A}$ expected at an open magnetopause generated by reconnection equatorward of the cusps. What processes other than reconnection equatorward of the cusps may contribute to the formation of the boundary layer? Entry of solar wind particles into the boundary layer due to curvature drift, gradient $B$ drift, and polarization drift always contribute to the formation of the boundary layer to some degree. However, Hill (1983) and Treumann and Baumjohann (1988) estimated that drift entry can only give a small percentage contribution. Impulsive penetration of irregularities in the solar wind containing excess momentum was suggested by Lemaire et al. (1985). Since we do not know of any method to elucidate the importance of impulsive penetration, this process is not considered in the present work.

In Sect. 6.3, we argued that cross-field diffusion caused by lower hybrid drift instability, gyroresonant pitch angle scattering, or kinetic Alfvén wave turbulence, based on the presently available theories, probably cannot transport solar wind plasma at a rate that would account for the thickness of the OBL. Moreover, and even more seriously, cross-field diffusion based solely on micro-instabilities should not form an OBL whose density profile shows a plateau inside the OBL, exhibiting a sharp step only at its inner edge, the transition to the inner boundary layer, as diffusive processes are restricted to gradient regions only. For diffusion to work here, one requires sufficiently steep gradients in temperature (i.e. pressure) or in the flows for which there are little indications in the data.

Magnetic reconnection with the interplanetary magnetic field directed northward (or nearly northward) is expected to occur poleward of the cusps. In the model of Le et al. (1996), the OBL for the northward interplanetary magnetic field consists of open flux tubes that have been reconnected at one of the two tail lobes. When these open flux tubes are recon- 
nected again at the other lobe, they become closed flux tubes and may form the IBL. On the dayside, the observed properties of the plasma in the OBL are entirely different from the properties of the plasma in the IBL (e.g. Sect. 5). In our opinion, it is hardly possible that the merging of open field lines and lobe field lines occurring poleward of the cusps at a distance of more than $15 R_{E}$ can effect the plasma seen on the dayside at low-latitudes drastically enough that its properties change from those seen in the OBL to those seen in the IBL. Step like profiles of the boundary layer with two plateaus (OBL and IBL) are also observed for directions of the interplanetary magnetic field different from northward and for high magnetic shear across the magnetopause, which does not support such a model.

According to Fig. 9 of Le et al. (1996), the plasma in the flux tubes forming the boundary layer is accelerated toward low-latitudes. The superposed epoch traces of the north-south component, $V_{p L}$, of the proton bulk velocity (Fig. 2) show that the plasma flow in the boundary layer is directed toward high-latitudes, both for low and high magnetic shear. This argues against the possibility that the low-latitude boundary layer is populated with solar wind plasma primarily from the cusps. At this point, it should be noted that we believe that reconnection poleward of the cusps occurs. It has been nicely demonstrated by Kessel et al. (1996) and Gosling et al. (1996). However, we doubt that this process is responsible for the formation of the low-latitude boundary layer on the dayside.

In summary, we do not find evidence that processes other than reconnection equatorward of the cusps play a major role in forming the dayside low-latitude boundary layer. The fact that most of the superposed epoch profiles look similar for the Walén events and non-Walén events suggests that the OBL is formed by the same process in both cases. In Paper I, we mentioned the possibility that the OBL is on open field lines that cross the magnetopause at a location farther away from the spacecraft. In this case, the solar wind plasma detected in the OBL may have entered along open field lines. If these field lines do not cross the magnetopause locally but rather farther away from the spacecraft, there is no reason why the observed local magnetopause should have the properties of a rotational discontinuity. Another possibility is that the flux tubes in the OBL have been first opened by reconnection, then filled with solar wind plasma, and have closed again later on (Nishida, 1989).

At this point, let us return to the superposed epoch profile of the magnetic pressure in Fig. 4. $P_{B}$ is constant across the magnetopause for Walén events, whereas it increases, on average, by a factor of two across the magnetopause for non-Walén events. The plasma in the vicinity of the magnetopause has $\beta \approx 1$ and pressure anisotropy $P_{\|} / P_{\perp} \approx 0.7$. Under these conditions, a change in $P_{B}$ by a factor of two is not possible according to the jump conditions at a rotational discontinuity (Hudson, 1970). Thus, the total magnetic flux observed in the OBL cannot cross the magnetopause locally. A large portion of the magnetic flux in the OBL must either be closed (maybe re-closed) or it crosses the magnetopause at a remote location.

\subsection{Formation of the inner boundary layer}

Warm electrons are a characteristic feature of the IBL. The flux of warm electrons parallel to $\boldsymbol{B}$ is almost always balanced by a flux antiparallel to $\boldsymbol{B}$. This balance was interpreted as evidence for closed field lines by Hall et al. (1991). On the other hand, the approximate balance of a field-aligned electron flux may also be observed for electrons on open field lines that are mirrored at low altitudes (Fuselier et al., 1997).

Let us discuss the possibility that the IBL is on newly opened field lines. When reconnection is in a steady state, velocity filtering occurs: solar wind particles entering the boundary layer do not fill the entire region of open field lines between the magnetopause and the separatrix, but only the region bounded by the magnetopause and an inner edge that depends on the field-aligned velocity, $v_{\|}^{\prime}$, of the particles in the de Hoffmann-Teller frame (e.g. Gosling et al., 1990). At the inner edge of particles with velocity $v_{\|}^{\prime}$, the time-of-flight of these particles from the magnetopause along a field line to the inner edge equals the time since the same field line was opened by reconnection. Since the particles did not have access to the boundary layer before the field line was opened, no particles are seen earthward of this inner edge or time-offlight boundary. If the transmitted solar wind ion population were a cool $\left(T_{p} \approx 0\right)$ beam, with all ions having the same velocity, $\boldsymbol{v} \approx \boldsymbol{V}_{p}$, the density of solar wind ions in the boundary layer would drop sharply at the time-of-flight boundary, defined by the field-aligned velocity, $v_{\|}^{\prime}$, of the beam in the de Hoffmann-Teller frame. Since the thermal velocity of the solar wind electrons is distinctly higher than $V_{p}$, they can enter the region between the inner edge of the ions and the separatrix of open and closed field lines. In reality, the thermal velocity of the ions is comparable to their bulk speed, $V_{p}$. Therefore, the time-of-flight boundary of the solar wind ions cannot be sharp. Furthermore, collective interactions prevent the particles from flying freely along $\boldsymbol{B}$. For example, the number of solar wind electrons in the region between the inner edge of the ions and the separatrix is limited by the action of the electrostatic field estabilished by the charge separation. In hybrid simulations (e.g. Lin and Lee, 1994) with ions treated as particles and electrons treated as a fluid, the inner edge of the solar wind ions is described as a slow mode structure consisting of a slow expansion fan and a slow mode shock.

The time-of-flight boundary of the solar wind ions is identified with the location of the strongest density gradient, i.e. with the interface between the OBL and the IBL. Since it is located sheathward of the separatrix between open and closed field lines, at least part of the IBL must be on newly opened field lines when reconnection is in a steady state. In this case, the presence of warm electrons in the IBL can be understood: they are those solar wind electrons that can enter the region between the time-of-flight boundary of the ions and the separatrix due to their high $\left|v_{\|}^{\prime}\right|$. The region located between the time-of-flight boundary and the separatrix is de- 
void of solar wind ions; no momentum has thus been transported from the magnetosheath to the plasma on the newly opened field lines of this region.

On 21 September 1984 the proton bulk flow changes both magnitude and direction at the interface between the OBL and the IBL (Paper I). $\boldsymbol{V}_{p}^{\prime}$ is field-aligned in the magnetosheath and in the OBL, it is not in the IBL whereas. This feature cannot be used to determine whether the IBL is on closed field lines or on newly opened field lines. Another important feature is the profile of the partial density, $N_{2 e}$, of electrons above $1.8 \mathrm{keV} . N_{2 e}$ has roughly the same value in the IBL and magnetosphere proper, whereas it drops at the interface between the IBL and the OBL. Since newly opened field lines also cross the OBL, the density of ring current electrons in an IBL on newly opened field lines should equal the density of ring current electrons in the OBL. Thus, the drop in $N_{2 e}$ indicates that most of the IBL observed on 21 September 1994 is on closed field lines and not on newly opened field lines.

The change in $\boldsymbol{V}_{p}^{\prime}$ and the drop in $N_{2 e}$ at the interface between the OBL and the IBL is confirmed by the superposed epoch analysis of all Walén events. The superposed epoch average of the angle between the proton bulk velocity in the de Hoffmann-Teller frame and the magnetic field (Fig. 6) changes from approximately $30^{\circ}$ in the OBL to almost $90^{\circ}$ in the IBL. The search for single particle signatures of open field lines reveals that on rare occasions, counterstreaming of solar wind ions and cold ions can be observed at the sheathward edge of the IBL. Farther earthward, particle signatures of open field lines are absent in the IBL. In particular, we never observe field-aligned streaming of ring current electrons. This supports the argument that most of the IBL is on closed field lines rather than on newly opened field lines. Near the interface between the OBL and the IBL, the superposed epoch profiles of the non-Walén events look similar to the superposed epoch profiles of the Walén events. For the non-Walén events we obviously do not observe steadystate reconnection. Therefore, the interface between the OBL and the IBL cannot be a time-of-flight boundary for the nonWalén events.

Reconnection may often not be in a steady state. Rather, it is time dependent or even turbulent. Evidence for time dependent patchy reconnection is provided by magnetopause crossings for which a particular particle signature changes its orientation in the course of the crossing from parallel to $\boldsymbol{B}$, to antiparallel to $\boldsymbol{B}$, or vice versa. Moreover, there are passes of the magnetopause region with multiple magnetopause crossings, where the test of the Walén relation indicates, $B_{n}<0$ for the first crossing, $B_{n}>0$ for the second crossing, and $B_{n}=0$ for the remaining crossings. Further evidence of time dependent patchy reconnection is provided by the occurrence of flux transfer events. The time dependent nature of reconnection can also be deduced from ground-based observations and satellite observations at low altitudes (e.g. Lockwood, 1995). Finally, evidence for time dependent, i.e. turbulent reconnection, could also be read from the enhanced magnetic wave spectra at around $1 \mathrm{~Hz}$ in the boundary layer. As we have discussed, such enhancements may be a sign of magnetic flux tube diffusion due to percolation (Galeev et al., 1986; LaBelle and Treumann, 1988; Belmont and Rezeau, 2001). Manifestation in a power law magnetic fluctuation spectrum at these frequencies could indicate that many spatial flux tube scales participate in this process, which naturally implies that there is interaction and reconnection between those flux tubes. Mixing at smaller scales and on the order of the ion gyroradius, therefore, necessarily causes slippage between the field and the plasma, and thus cuases a violation of the frozen-in condition.

If a pulse of reconnection is followed by a period without local reconnection, the time-of-flight boundary of the solar wind ions propagates toward the terrestrial end of an open flux tube and is no longer visible near the equatorial plane. What is seen near the equatorial plane is a bundle of open flux tubes that has been produced by the pulse of reconnection and that is now separated from the closed flux tubes by a topological boundary, across which the plasma density drops from values comparable to the magnetosheath density to values comparable to the magnetospheric density. If the interface between the OBL and the IBL is interpreted as such a topological boundary between open and closed field lines, it can be understood why $N_{2 e}$ drops at this interface and why single particle signatures of open field lines are absent in the IBL.

For all crossings in our data set, solar wind ions are detected at least in part of the IBL. In Sect. 7.1, we gave arguments why reconnection poleward of the cusps cannot be responsible for the formation of the boundary layer on the dayside. Reconnection equatorward of the cusps cannot easily explain the presence of solar wind ions in the IBL: there should be no solar wind ions earthward of the topological boundary between open and closed field lines and there should not be solar wind ions earthward of their own timeof-flight boundary. According to Sect. 6.3, estimated microscopic diffusion coefficients do not account for the formation of the IBL. We must, however, admit that the diffusion process itself is barely understood at the present time. Our estimates were based on the assumption of anomalous transport due to micro-instabilities and are thus subject to the criticism as to what degree we know the evolution and cascade of such instabilities. Possibly, localization in small patches causes much stronger diffusivities than estimated by us. Furthermore, one important energy source for transport can be found in the intense spectrum of low frequency magnetosheath fluctuations (Johnson and Cheng, 1997; Belmont and Rezeau, 2001). Their contribution to diffusion and reconnection is beginning to be understood and is very promising, as it might lead to a deeper understanding of the entire turbulent process of reconnection and the reconnective self-organization at the magnetopause. Finally, the concept of micro-diffusion has to be combined with macroscopic eddy motions. Whenever these come into play, micro-diffusion needs to act only over short spatial scales, while large eddy non-diffusive motion transports the plasma to the locations where it changes the identity of a flux tube. These processes are not yet under- 
stood and need further investigation. Formation of the IBL, in our view, thus still remains barely understood (see also our above comments on turbulent reconnection).

We conclude by mentioning that another possible, though unconventional, way out of this dilemma would be that the IBL is not formed on the dayside, but by processes operating farther down in the magnetotail. In Sect. 5.3, we argued that the dawn-dusk component of the proton bulk velocity in the IBL and magnetosphere on the evening side is directed from the nightside toward local noon (Fig. 7). So it is indeed possible that the processes forming the IBL operate farther tailward of the region examined in this work. If this is true, the terms "halo" and "mixing region" coined by Skopke et al. (1981) and Fujimoto et al. (1998a) are more adequate than the term "inner boundary layer". Fujimoto et al. (1998a) suggested that the solar wind particles in the mixing region might be supplied to the magnetosphere by the same process as the solar wind particles seen in the cold dense plasma sheet of the tail. The IBL, in such a case, appears to be nothing else but the dayside extension of the near-Earth edge of the tailward plasma sheet.

Acknowledgement. We thank H. Lühr and B. Häusler for making available the magnetic field and wave data, respectively. G. Paschmann was the principal investigator of the AMPTE/IRM plasma instrument. We thank Norbert Skopke for fruitful discussions and will remember him as a wonderful person. We acknowledge with thanks the helpful comments of the two referees, in particular their clarification of the connection between micro- and macro-diffusivities and pointing out the relevance of the papers by Rezeau and Belmont (2001) and Belmont and Rezeau (2001) to the present investigation.

Topical Editor G. Chanteur thanks L. Rezeau and another referee for their help in evaluating this paper.

\section{References}

Anderson, B. J., Fuselier, S. A., and Murr, D., Electromagnetic ion cyclotron waves observed in the plasma depletion layer, Geophys. Res. Lett., 18, 1955-1958, 1991.

Anderson, R. R., Harvey, C. C., Hoppe, M. M., Tsurutani, B. T., Eastman, T. E., and Etcheto, J., Plasma waves near the magnetopause, J. Geophys. Res., 87, 2087-2107, 1982.

Bauer, T. M., Baumjohann, W., Treumann, R. A., Skopke, N., and Lühr, H., Low-frequency waves in the near-Earth plasma sheet, J. Geophys. Res., 100, 9605-9617, 1995.

Bauer, T. M., Paschmann, G., Skopke, N., Treumann, R. A., Baumjohann, W., and Phan, T.-D., Fluid and particle signatures of dayside reconnection, Ann. Geophysicæ, submitted, 2000.

Baumjohann, W. and Treumann, R. A., Basic Space Plasma Physics, Imperial College Press, London, 1996.

Belmont, G., Reberac, F., and Rezeau, L. , Resonant amplification of magnetosheath MHD fluctuations at the magnetopause, Geophys. Res. Lett., 22, 295-298, 1995.

Belmont, G. and Rezeau, L., Magnetopasue reconnection induced by magnetosheath Hall-MHD fluctuations, J. Geophys. Res., 106, 10751-10 760, 2001.

Boehm, M. H., Clemmons, J., Wahlund, J.-E., Eriksson, A., Eliasson, L., Blomberg, L., Kintner, P., and Höfner, H., Observations of an upward-directed electron beam with the perpendicular temperature of the cold ionosphere, Geophys. Res. Lett., 22, 21032106, 1995.

Borovsky, J. E., Thomsen, M. F., and McComas, D. J., The superdense plasma sheet: Plasmaspheric origin, solar wind origin, or ionospheric origin?, J. Geophys. Res., 102, 22 089-22 097, 1997.

Carlson, C. W., Mc Fadden, J. P., Ergun, R. E., Temerin, M., Peria, W., Moser, F. S., Klumpar, D. M., Shelley, E. G., Peterson, W. K., Moebius, E., Elphic, R., Strangeway, R., Cattell, C., and Pfaff, R., FAST observations in the downward auroral current region: Energetic upgoing electron beams, parallel potential drops, and ion heating, Geophys. Res. Lett., 25, 2017-2020, 1998.

Denton, R. E., Gary, S. P., Li, X., Anderson, B. J., LaBelle, J. W., and Lessard, M., Low-frequency waves in the magnetosheath near the magnetopause, J. Geophys. Res., 100, 5665-5679, 1995.

Elphic, R. C., Weiss, L. A., Thomsen, M. F., and McComas, D. J., Evolution of plasmaspheric ions at geosynchronous orbit during times of high geomagnetic activity, Geophys. Res. Lett., 23, 2189-2192, 1996.

Fairfield, D. H., Average and unusual locations of the Earth's magnetopause and bow shock, J. Geophys. Res., 76, 6700-6716, 1971.

Fujimoto, M., Mukai, T., Kawano, H., Nakamura, M., Nishida, A., Saito, Y., Yamamoto, T., and Kokubun, S., Structure of the lowlatitude boundary layer: A case study with Geotail data, J. Geophys. Res., 103, 2297-2308, 1998a.

Fujimoto, M., Teresawa, T., Mukai, T., Saito, Y., Yamamoto, T., and Kokubun, S., Plasma entry from the flanks of the near-Earth magnetotail: Geotail observations, J. Geophys. Res., 103, 22972308, 1998b.

Fuselier, S. A., Klumpar, D. M., Peterson, W. K., and Shelley, E. G., Direct injection of ionospheric $\mathrm{O}^{+}$into the dayside low latitude boundary layer, Geophys. Res. Lett., 16, 1121-1124, 1989.

Fuselier, S. A., Klumpar, D. M., and Shelley, E. G., Ion reflection and transmission during reconnection at the Earth's subsolar magnetopause, Geophys. Res. Lett., 18, 139-142, 1991.

Fuselier, S. A., Anderson, B. J., and Onsager, T. G., Electron and ion signatures of field line topology at the low shear magnetopause, J. Geophys. Res., 102, 4847-4863, 1997.

Galeev, A. A., Kuzneetsova, M. M., and Zeleny, L. M., Magnetopause stability threshold for patchy reconnection, Space Sci. Rev., 44, 1-41, 1986.

Gosling, J. T., Thomsen, M. F., Bame, S. J., Onsager, T. G., and Russell, C. T., The electron edge of the low latitude boundary layer during accelerated flow events, Geophys. Res. Lett., 17, 18331836, 1990.

Gosling, J. T., Thomsen, M. F., Le, G., and Russell, C. T., Observations of magnetic reconnection at the lobe magnetopause, J. Geophys. Res., 101, 24 765-24773, 1996.

Gurnett, D. A., Anderson, R. R., Tsurutani, B. T., Smith, E. J., Paschmann, G., Haerendel, G., Bame, S. J., and Russell, C. T., Plasma wave turbulence at the magnetopause: Observations from ISEE 1 and 2, J. Geophys. Res., 84, 7043-7058, 1979.

Hall, D. S., Chaloner, C. P., Bryant, D. A., Lepine, D. A., and Tritakis, V. P., Electrons in the boundary layers near the dayside magnetopause J. Geophys. Res., 96, 7869-7891, 1991.

Hapgood, M. A. and Bryant, D. A., Re-ordered electron data in the low-latitude boundary layer, Geophys. Res. Lett., 17, 20432046, 1990.

Häusler, B., Anderson, R. R., Gurnett, D. A., Koons, H. C., Holzworth, R. H., Bauer, O. H., Treumann, R., Gnaiger, K., Odem, D., Harbridge, W. B., and Eberl, F., The plasma wave instrument 
on board the IRM satellite, IEEE Trans. Geosci. Remote Sens., GE-23, 267-273, 1985.

Hill, T. W., Solar-wind magnetosphere coupling, in Solar-Terrestrial Physics, edited by Carovillano, R. L. and Forbes, J. M., pp. 261302, D. Reidel, Dordrecht, 1983.

Hudson, P. D., Discontinuities in an anisotropic plasma and their identification in the solar wind, Planet. Space Sci., 18, 16111622,1970

Hultqvist, B., Oieroset, M., Paschmann, G., and Treumann, R. A. (eds.), Magnetospheric Plasma Sources and Losses, Space Science Series of ISSI, vol. 6, Chapter 5, Kluwer Acad. Publ., Dordrecht, pp. 207-283, 1999.

Johnson, J. R. and Cheng, C. Z., Kinetic Alfvén waves and plasma transport at the magnetopause, Geophys. Res. Lett., 24, 14231426, 1997.

Kessel, R. L., Chen, S.-H., Green, J. L., Fung, S. F., Boardsen, S. A., Tan, L. C., Eastman, T. E., Craven, J. D., and Frank, L. A., Evidence of high-latitude reconnection during northward IMF: Hawkeye observations, Geophys. Res. Lett., 23, 583-586, 1996.

LaBelle, J. and Treumann, R. A., Plasmawaves at the dayside magnetopause, Space Sci. Rev., 47, 175-208, 1988.

LaBelle, J., Treumann, R. A., Haerendel, G., Bauer, O. H., Paschmann, G., Baumjohann, W., Lühr, H., Anderson, R. R., Koons, H. C., and Holzworth, R. H., AMPTE IRM observations of waves associated with flux transfer events in the magnetosphere, J. Geophys. Res., 92, 5827-5843, 1987.

Le, G., Russell, C. T., Gosling, J. T., and Thomsen, M. F., ISEE observations of low-latitude boundary layer for northward interplanetary magnetic field: Implications for cusp reconnection, J. Geophys. Res., 101, 27 239-27 249, 1996.

Lee, L. C., Johnson, J. R., and Ma, Z. W., Kinetic Alfvén waves as a source of plasma transport at the dayside magnetopause, $\mathrm{J}$. Geophys. Res., 100, 17 405-17 411, 1994.

Lemaire, J., Plasmoid motion across a tangential discontinuity (with application to the magnetopause), J. Plasma Phys., 33, 425-436, 1985.

Lin, Y. and Lee, L. C., Structure of reconnection layers in the magnetosphere, Space Sci. Rev., 65, 59-179, 1994.

Lockwood, M., Ground-based and satellite observations of the cusp: Evidence for pulsed magnetopause reconnection, in Physics of the Magnetopause, Geophys. Monogr. Ser., vol. 90, edited by B. U. Ö. Sonnerup et al., pp. 417-426, AGU, Washington, D.C., 1995.

Lockwood, M., Relationship of dayside auroral precipitations to the open-closed separatrix and the pattern of convective flow, J. Geophys. Res., 102, 17 475-17 487, 1997.

Lotko, W. and Sonnerup, B. U. Ö., The low-latitude boundary layer on closed field lines, in Physics of the Magnetopause, Geophys. Monogr. Ser., vol. 90, edited by Sonnerup, B. U. Ö., et al., pp. 371-386, AGU, Washington, D. C., 1995.

Lundin, R., Eliasson, L., Hultqvist, B., and Stasiewicz, K., Plasma energization on auroral field lines as observed by the VIKING spacecraft, Geophys. Res. Lett., 14, 443-446, 1987.

Lühr, H., Klöcker, N., Oelschlägel, W., Häusler, B., and Acuña, M., The IRM fluxgate magnetometer, IEEE Trans. Geosci. Remote Sens., GE-23, 259-261, 1985.

Nakamura, M., Fujimoto, M., Kawano, H., Mukai, T., Saito, Y., Yamamoto, T., Tsurada, K., Teresawa, T., and Kokubun, S., Geotail observations at the dayside magnetopause - Confirmation of reconnection events, Adv. Space Res., 20(4-5), 779-788, 2000.

Nishida, A., Can random reconnection on the magnetopause produce the low latitude boundary layer?, Geophys. Res. Lett., 16,
227-230, 1989.

Ogilvie, K. W., Fitzenreiter, R. J., and Scudder, J. D., Observations of electron beams in the low latitude boundary layer, J. Geophys. Res., 89, 10723-10 732, 1984.

Paschmann, G., Loidl, H., Obermayer, P., Ertl, M., Laborenz, R., Skopke, N., Baumjohann, W., Carlson, C. W., and Curtis, D. W., The plasma instrument for AMPTE/IRM, IEEE Trans. Geosci. Remote Sens., GE-23, 262-266, 1985.

Paschmann, G., Papamastorakis, I., Baumjohann, W., Skopke, N., Carlson, C. W., Sonnerup, B. U. Ö., and Lühr, H., The magnetopause for large magnetic shear: AMPTE/IRM observations, J. Geophys. Res., 91, 11 099-11 115, 1986.

Paschmann, G., Baumjohann, W., Skopke, N., Phan, T.-D., and Lühr, H., Structure of the dayside magnetopause for low magnetic shear, J. Geophys. Res., bf 98, 13 409-13 422, 1993.

Phan, T.-D. and Paschmann, G., Low-latitude dayside magnetopause and boundary layer for high magnetic shear: 1. Structure and motion, J. Geophys. Res., 101, 7801-7815, 1996.

Phan, T.-D., Paschmann, G., Baumjohann, W., Skopke, N., and Lühr, H., The magnetosheath region adjacent to the dayside magnetopause: AMPTE/IRM observations, J. Geophys. Res., 99, 121-141, 1994.

Phan, T.-D., Paschmann, G., and Sonnerup, B. U. Ö., Low-latitude dayside magnetopause and boundary layer for high magnetic shear: 2. Occurrence of magnetic reconnection, J. Geophys. Res., 101, 7817-7828, 1996.

Phan, T.-D., Kistler, L. M., Klecker, B., Haerendel, G., Paschmann, G., Sonnerup, B. U. Ö., Baumjohann, W., Bavassano-Cattaneo, M. B., Carlson, C. W., DiLellis, A. M., Fornacon, K.-H., Frank, L. A., Fujimoto, M., Georgescu, E., Kokubun, S., Moebius, E., Mukai, T., Øieroset, M., Paterson, W. R., and Reme, H., Extended magnetic reconnection at the Earth's magnetopause from detection of bi-directional jets, Nature, 404, 848-850, 2000.

Pottelette, R. and Treumann, R. A., Impulsive broadband electrostatic noise in the cleft: A signature of dayside reconnection, J. Geophys. Res., 103, 9299-9308, 1998.

Rezeau, L. and Belmont, G., Magnetic turbulence at the magnetopause, a key problem for understanding the solar wind/magnetosphere exchanges, Space Sci. Rev., 95, 427-441, 2001.

Rezeau, L., Roux, A., and Russell, C. T., Characterization of smallscale structures at the magnetopause from ISEE measurements, J. Geophys. Res., 98, 179, 1993.

Rosenbluth, M. N., Sagdeev, R. Z., Taylor, J. B., and Zaslavsky, G. M., The destruction of magnetic surfaces, Nucl. Fusion, 6, 297 300, 1966.

Russell, C. T. and Elphic, R. C., ISEE observations of flux transfer events at the dayside magnetopause, Geophys. Res. Lett., 6, 3336,1979

Sagdeev, R. Z., The 1976 Oppenheimer lectures: Critical problems in plasma astrophysics. I. Turbulence and nonlinear waves, Rev. Mod. Phys., 51, 1-9, 1979.

Sazhin, S. S., Walker, S. N., and Woolliscroft, L. J. C., Observations and theory of whistler-mode waves in the vicinity of the Earth's magnetopause, Adv. Space Res., 11, (9), 33-36, 1991.

Skopke, N., Paschmann, G., Haerendel, G., Sonnerup, B. U. Ö., Bame, S. J., Forbes, T. G., Hones, Jr., E. W., and Russell, C. T., Structure of the low latitude boundary layer, J. Geophys. Res., 86, 2099-2110, 1981.

Scudder, J. D., Puhl-Quinn, P. A., Mozer, F. S., Ogilvie, K. W., and Russell, C. T., Generalized Walén tests through Alfvén waves and rotational discontinuities using electron flow velocities, J. 
Geophys. Res., 104, 19 817-19 833, 1999.

Song, P., Elphic, R. C., Russell, C. T., Gosling, J. T., and Cattell, C. A., Structure and properties of the subsolar magnetopause for northward IMF: ISEE observations, J. Geophys. Res., 95, 63756387, 1990.

Song, P., Russell, C. T., Fitzenreiter, R. J., Gosling, J. T., Thomsen, M. F., Mitchell, D. G., Fuselier, S. A., Parks, G. K., Anderson, R. R., and Hubert, D., Structure and properties of the subsolar magnetopause for northward interplanetary magnetic field: Multiple-instrument particle observations, J. Geophys. Res., 98, $11319-11337,1993$.

Sonnerup, B. U. Ö., Paschmann, G., Papamastorakis, I., Skopke, N., Haerendel, G., Bame, S. J., Asbridge, J. R., Gosling, J. T., and Russell, C. T., Evidence for magnetic field reconnection at the Earth's magnetopause, J. Geophys. Res., 86, 10 049-10 067, 1981.

Sonnerup, B. U. Ö., Papamastorakis, I., Paschmann, G., and Lühr, H., The magnetopause for large magnetic shear: Analysis of convection electric fields from AMPTE/IRM, J. Geophys. Res., 95, 10 541-10 557, 1990.

Southwood, D. J., Some features of field line resonances in the magnetosphere, Planet. Space Sci., 22, 483-491, 1974.

Streltsov, A. and Lotko, W., The fine structure of dispersive, nonradiative field line resonance layers, J. Geophys. Res., 101, 5343-5355, 1996.

Thorne, R. M. and Tsurutani, B. T., Wave-particle interactions in the magnetopause boundary layer, in Physics of Space Plasmas (1990), SPI Conf. Proc. Rep. Ser., vol. 10, edited by Chang, T. S., pp. 119-150, Scientific Publishers, Cambridge, Mass., 1991.

Treumann, R. A., Theory of super-diffusion for the magnetopause, Geophys. Res. Lett., 24, 1727-1730, 1997.
Treumann, R. A. and Baumjohann, W., Particle trapping at a tangential discontinuity: Multiple incidence, Planet. Space Sci., 36, 1477-1491, 1988.

Treumann, R. A., LaBelle, J., Haerendel, G., and Pottelette, R., Anomalous plasma diffusion and the magnetopause boundary layer, IEEE Trans. Plasma Sci., 20, 833-842, 1992a.

Treumann, R. A., LaBelle, J., and Pottelette, R., Plasma transport through magnetic boundaries, in Proc. 26th ESLAB Symp., ESA-SP-346, 115-126, Paris, 1992b.

Treumann, R. A., LaBelle, J., and Bauer, T. M., Diffusion processes: An observational perspective, in Physics of the Magnetopause, Geophys. Monogr. Ser., vol. 90, edited by Sonnerup, B. U. Ö., et al., pp. 331-341, AGU, Washington, D. C., 1995.

Treumann, R. A. and Skopke, N., Diffusive processes, Space Sci. Rev., 88, 389-404, 1999.

Tsurutani, B. T. and Smith, E. J., Two types of magnetospheric ELF chorus and their substorm dependences, J. Geophys. Res., 82, 5112-5128, 1977.

Winske, D. and Omidi, N., Diffusion at the magnetopause: Hybrid simulations, J. Geophys. Res., 100, 11 923-11 933, 1995.

Winske, D., Thomas, V. A., and Omidi, N., Diffusion at the magnetopause: A theoretical perspective, in Physics of the Magnetopause, Geophys. Monogr. Ser., vol. 90, edited by Sonnerup, B. U. Ö., et al., pp. 321-330, AGU, Washington, D. C., 1995.

Zhu, X. and Kivelson, M. G., Analytic formulation and quantitative solutions of the coupled ULF wave problem, J. Geophys. Res., 93, 8602-8622, 1988.

Zhu, X. and Kivelson, M. G., Global mode ULF pulsations in a magnetosphere with non-monotonic Alfvén velocity profile, J. Geophys. Res., 94, 1479-1485, 1989. 